\title{
Les pensions de retraite au Canada : les inégalités d'un système dualiste
}

\section{THE CASUALTIES OF CANADA'S DUALIST PENSION POLICY LAS PENSIONES DE JUBILACIÓN EN CANADA : LAS DESIGUALDADES CAUSADAS POR UN SISTEMA DUALISTA}

\section{Lynn McDonald}

Volume 24, numéro 2, automne 1995

Activité et retraite

URI : https://id.erudit.org/iderudit/010188ar

DOI : https://doi.org/10.7202/010188ar

Aller au sommaire du numéro

Éditeur(s)

Association des démographes du Québec

ISSN

0380-1721 (imprimé)

1705-1495 (numérique)

Découvrir la revue

Citer cet article

McDonald, L. (1995). Les pensions de retraite au Canada : les inégalités d'un système dualiste. Cahiers québécois de démographie, 24(2), 205-244.

https://doi.org/10.7202/010188ar
Résumé de l'article

À cause de leur caractère à la fois public et semi-privé, les politiques sociales canadiennes ont engendré un système dualiste de sécurité du revenu pour les retraités. Certains sont entièrement tributaires des régimes de retraite publics tandis que d'autres bénéficient à la fois de régimes publics et de régimes privés. Cette division se manifeste dans leurs conditions de vie, qui ne sont pas les mêmes suivant qu'ils travaillaient dans le secteur central ou dans le secteur périphérique de l'économie. À l'aide de données provenant de l'Enquête sur le vieillissement et l'autonomie, l'auteur étudie les effets du dualisme économique sur le revenu de retraite des hommes et des femmes de son échantillon $(\mathrm{N}=20036)$. Elle compare les retraités du centre et de la périphérie au point de vue de leurs caractéristiques socio-démographiques et de diverses caractéristiques relatives au travail, au revenu et aux dispositions prises pour la retraite. Des modèles de régression lui permettent ensuite de déterminer les facteurs qui influencent le revenu des retraités du centre et de la périphérie. Les résultats tendent à montrer que le système canadien de pensions de retraite accentue la précarité économique des femmes retraitées, dont on sait qu'elle est liée à leur situation sur le marché du travail et à leurs responsabilités familiales. Ce régime dualiste contribue à créer une sous-classe de retraités, et ses principales victimes sont les femmes qui ont passé leur vie active dans la périphérie de l'économie. 
Cahiers québécois de démographie

Vol. 24, no 2, automne 1995, p. 205-244.

\title{
Les pensions de retraite au Canada : les inégalités d'un système dualiste
}

\author{
Lynn MCDONALD *
}

Le débat sur la crise de l'État providence qui se poursuit un peu partout au Canada depuis la fin des années 1970 n'a guère dépassé le niveau des considérations académiques (McDaniel et Gee, 1993; Myles et Teichroew, 1991), jusqu'à ce que l'ampleur du déficit $(37,9$ milliards de dollars) et l'envergure de la dette projetée (550,7 milliards de dollars) du pays pour l'année 19941995 fassent monter le ton du débat socio-politique. L'évidence du lien entre le vieillissement démographique et ce contexte budgétaire n'a pas manqué de projeter les travailleurs vieillissants et les retraités à l'avant-scène de l'actualité, et de plus en plus on attend d'eux une contribution accrue à la réduction du déficit ${ }^{1}$. La prétendue amélioration de la situation socioéconomique des personnes âgées au Canada et le fardeau déraisonnable que représentera l'augmentation de leur nombre pour les régimes de retraite publics servent de justification à cette exigence, ainsi qu'à la promesse explicite du gouvernement fédéral de réviser entièrement ses politiques en matière de retraite en 1997. Le Canada, à l'instar d'un grand nombre de pays industrialisés, s'apprête à réformer en profondeur son système de protection sociale, et les régimes de retraite lui serviront de banc d'essai dans son entreprise de restructuration du filet de sécurité (Myles et Teichroew, 1991).

Après avoir encouragé la retraite précoce tout au long des années 1980 afin de réduire le chômage, beaucoup de pays

\footnotetext{
* Faculté de service social, Université de Toronto. Communication présentée au International Sociological Association Research on Ageing Intercongress Meeting (Melbourne, Australie, 24-26 avril 1995). Traduction : Johanne Archambault, sous la supervision d'Yves Carrière.

1 Les pensions versées aux personnes ãgées totalisent plus de vingt milliards de dollars.
} 
industrialisés d'Occident en sont venus à la conclusion que l'ascension vertigineuse des coûts des régimes de retraite publics posait un problème plus grave, et ils ont pris des mesures pour contrer le mouvement. Les trois principales stratégies ont consisté à élever l'âge de la retraite, comme en Allemagne, aux États-Unis et en Italie (Sheppard, 1991; Guillemard et Rein, 1993; Chen, 1994), à rendre plus flexible le processus de prise de retraite et à implanter le partage du temps de travail, comme en France, en Allemagne et au Canada (Lussier et Wister, 1995; Laczko et Phillipson, 1991), et enfin à transférer le fardeau des coûts du secteur public au secteur privé ou semi-privé, comme au Canada, aux États-Unis et en GrandeBretagne (Casey, 1989; Laczko et Phillipson, 1991; Walker, 1991; Myles et Teichroew, 1991; Guillemard et Rein, 1993; Hardy et al., 1993).

Dans bien des pays, ce transfert des coûts implique un nouveau partage des rôles entre les gouvernements et le secteur privé dans le domaine de la sécurité sociale et l'adoption d'un modèle où l'assistance sociale est strictement supplétive par rapport au marché et à la famille (Guest, 1990). Ce "modèle supplétif" vise à donner un revenu de subsistance aux travailleurs pauvres (une aide sociale), plutôt que d'assurer à l'ensemble des travailleurs un revenu suffisant tout au long de leur retraite (comme le ferait une politique d'assurance sociale). Au Canada, la transition vers l'approche supplétive est devenue manifeste durant les années 1980, quand on a commencé à réclamer aux personnes âgées jouissant d'une certaine aisance le remboursement de sommes reçues en vertu du programme de sécurité de la vieillesse. En substance, cela revenait à mettre de côté la notion d'universalité pour consacrer aux plus pauvres les sommes ainsi rendues disponibles. Simultanément, on a haussé le niveau des cotisations autorisées aux régimes enregistrés d'épargne-retraite et aux régimes de retraite liés à l'emploi, élargissant d'autant le rôle du secteur privé dans le champ de la protection sociale ${ }^{2}$.

C'est essentiellement ce type de partage des rôles entre l'État et le secteur semi-privé en matière de sécurité du revenu qui explique le caractère dualiste de notre système de pensions, et

2 Tout en réduisant la valeur absolue des régimes enregistrés d'épargneretraite et des régimes de retraite agréés pour les années 1996 et 1995 , le budget fédéral de 1995 prévoit que les cotisations autorisées pourront augmenter de 1000 dollars par an, jusqu'en 1999 dans le cas des REER, jusqu'en 1998 dans le cas des régimes de retraite agréés. 
des chercheurs se sont employés à en dégager les conséquences pour les revenus de retraite des Canadiens (Schellenberg, 1994; Myles et Teichroew, 1991). Myles et Teichroew (1991) notent que certains travailleurs âgés ne touchent que la pension versée par le régime public, tandis que d'autres "bénéficient de régimes de retraite liés à l'emploi et de régimes d'épargneretraite gérés par le secteur privé mais subventionnés par l'État» (p. 92) ${ }^{3}$. De l'avis de certains, cette dualité du système de pensions, telle qu'elle se manifeste dans le revenu de retraite des Canadiens, reproduit la division structurelle de l'économie que décrivent les théories de la segmentation du marché du travail et du dualisme économique (ibid.).

Un modèle structurel d'explication du revenu de retraite basé sur la théorie du dualisme économique montrerait que la pauvreté des personnes âgées retraitées n'est pas simplement l'expression de l'échec individuel pointé du doigt par les tenants du rôle purement supplétif de l'État en matière de protection sociale, mais résulte de l'inégalité des chances inhérente à la structure de l'économie. Et surtout, un tel modèle ferait valoir que toute addition aux responsabilités de l'individu eu égard à sa propre sécurité financière améliorerait encore davantage la situation des gens qui sont déjà favorisés et aggraverait les disparités de revenu entre les personnes âgées. Ce problème revêt un caractère d'urgence car le gouvernement fédéral, endossant l'approche supplétive, maintient le cap sur des politiques d'incitation des travailleurs à prendre eux-mêmes les moyens d'avoir de quoi vivre au moment de leur retraite. Il importe de savoir qui souffrira le plus de cette tendance et comment ses effets se manifesteront.

Le but de cette recherche est donc d'examiner l'effet du secteur économique - le centre et la périphérie de l'économie sur le revenu de retraite des Canadiens afin de déterminer si la division des retraités en deux groupes eu égard aux sources de leurs revenus de retraite correspond à la division de l'économie définie par la théorie du dualisme économique. Nous faisons l'hypothèse que les retraités du centre sont plus susceptibles de bénéficier à la fois du régime public et des régimes semi-privés que les travailleurs de la périphérie, et que le revenu de retraite de ces derniers risque fort de provenir intégralement du régime

3 Les régimes de retraite liès à l'emploi et les régimes d'épargne-retraite agréés sont subventionnés par des diminutions d'impôt associées aux cotisations versées et aux gains accumulés, si bien que ces régimes privés pourraient être qualifiés, plus justement, de "semi-privés". 
public. Si nos résultats confirment cette hypothèse, les travailleurs de la périphérie sont ceux qui souffriraient le plus de la mise en œuvre de mesures affaiblissant le régime public de pensions. À l'aide de l'Enquête sur le vieillissement et l'autonomie, menée en septembre 1991, nous comparerons les travailleurs du centre et ceux de la périphérie au point de vue de certaines caractéristiques socio-démographiques, du travail, du revenu, de la retraite et de la préparation financière de la retraite. L'estimation des modèles devrait faire ressortir les facteurs qui influencent le revenu des retraités selon le secteur économique et le sexe.

\section{LA THÉORIE DU DUALISME ÉCONOMIgUE}

La théorie du dualisme économique découle de la théorie de la segmentation du marché du travail, issue des travaux de théoriciens marxistes qui ont discerné, au sein des sociêtés capitalistes, le développement de deux grands secteurs économiques distincts (Krahn et Lowe, 1993). Elle s'oppose aux théories économiques néo-classiques et au rôle qu'elles accordent à la notion de capital humain. Selon ces théories, le travailleur est un individu rationnel qui investit dans sa formation et s'efforce de maximiser le rendement économique de cet investissement; la libre concurrence entre les firmes désireuses d'acquérir les habiletés des divers types de travailleurs assure à ces derniers une rémunération théoriquement égale à leur productivité (Becker, 1975; Thurow, 1975; Sørensen et Kalleberg, 1981; Sakamoto et Chen, 1991). Ainsi la réussite socio-économique du travailleur est-elle directement liée à ce qu'il apporte sur le marché, et le revenu dont il jouit au moment de sa retraite se situe dans le prolongement de ce processus (Henretta et Campbell, 1976; Schulz, 1980). La théorie du capital humain suit la même logique que le modèle du rôle supplétif de l'État en matière de protection sociale : chacun est responsable de soi.

Les théoriciens du dualisme économique ont voulu démonter les mécanismes structurels de ce processus. Selon eux, à mesure que le capitalisme concurrentiel a cédé le pas au capitalisme monopolistique, le marché s'est segmenté en deux grands secteurs généralement appelés "centre" et "périphérie" (Beck, Horan et Tolbert, 1978). Les secteurs industriels qui constituent le centre sont dominés par de grandes sociétés actives dans la fabrication de biens durables, les industries de la construction, les mines, la pétrochimie, le transport, les 
communications, certains types de commerce de gros et certaines activités professionnelles. Les firmes du centre se caractérisent par une productivité élevée, des profits importants, une forte intensité en capital, un caractère souvent oligopolistique et un taux de syndicalisation généralement élevé. Pour assurer leur pérennité, ces organisations accordent des salaires et des conditions de travail supérieurs à ceux qui existent ailleurs, offrent des possibilités de formation à leurs employés et leur procurent des avantages sociaux intéressants (Nelson, 1994). On peut y poursuivre une carrière bien réglée, donnant accès à une rémunération et à un prestige professionnel en progression continue (Spilerman, 1977), et débouchant normalement sur une situation économique tout à fait avantageuse au moment de la retraite. En principe, le secteur étatique s'apparente au secteur central (Averitt, 1968). Au sein du secteur central, certains chercheurs distinguent également entre personnel autonome et personnel subordonné (Piore, 1975; Edwards, 1979). Cette dernière catégorie correspond surtout aux cols-bleus syndiqués, la première aux gestionnaires, professionnels et techniciens jouissant de perspectives d'avancement et de rémunération plus intéressantes.

À la périphérie de l'économie se trouvent les petites firmes, actives surtout dans l'agriculture, dans la fabrication de certains biens durables, dans certains types de commerce de gros et de détail et dans la plupart des services. Ces petites firmes peuvent rendre des services de niveau inférieur (restaurants, magasins de quartier...) aussi bien que de niveau plus élevé (fournisseurs de matériel de bureau par exemple), voire produire des biens de niveau supérieur. En général, elles ne contrôlent pas les marchés sur lesquels elles écoulent leurs produits, où la concurrence est vive, et leurs marges de profit sont plutôt minces. Elles visent principalement à maximiser leur rendement à court terme car elles sont plus vulnérables aux cycles économiques et il leur est difficile de faire absorber leurs augmentations de coûts de main-d'œuvre par leurs clients. Le secteur périphérique est intensif en main-d'œuvre, peu productif, fait appel à des travailleurs faiblement qualifiés, n'offre pas beaucoup de formation à ses employés et les syndicats y sont peu présents (Reiter, 1991). Ces facteurs se traduisent par des salaires et des avantages sociaux ininteressants, des cheminements de carrière imprévisibles et, logiquement, une situation économique moins privilégiée pour les retraités de la périphérie. 
En gérontologie, le dualisme économique et son application à la retraite sont habituellement abordés dans la perspective de l'économie politique. Certains chercheurs nord-américains l'étudient à l'échelle macrosociale (Schulz, 1991; Minkler et Estes, 1991; Quadagno et Myles, 1991), centrant leurs analyses de la retraite sur les liens entre politiques gouvernementales, classes sociales et économie (McDonald et Wanner, 1990). James Dowd (1980), qui a probablement été le premier Nordaméricain à faire appel à l'économie politique pour étudier le dualisme économique, a postulé que le secteur économique où travaille l'individu conditionne sa retraite future, notamment en ce qui concerne le niveau de revenu dont il jouira.

Les Européens, dans une conjoncture socio-économique et politique différente, ont tenté d'associer les perspectives de l'économie politique et de la théorie du cycle de vie pour bâtir une théorie de la retraite. Essentiellement, ils essaient de voir comment l'effet combiné des politiques d'assistance sociale, des politiques de retraite, de la structure des marchés du travail ainsi que de la culture façonne le cycle de vie. Ils définissent la retraite comme la fin ou le début de la fin de la vie active. Loin d'être fixes, le seuil et le contenu de ce stade de la vie dépendent de l'évolution des régimes de retraite et du système de protection sociale en général, de la conjoncture économique, des valeurs ambiantes, des comportements individuels et collectifs des retraités et des grands événements historiques (Guillemard et Rein, 1993). Guillemard (1991 : 219), par exemple, est d'avis que les régimes de retraite n'assurent plus la régulation des retraites précoces en Europe mais que, plutôt, les rentes d'invalidité protègent les individus entre le moment où ils quittent le marché du travail et le moment où ils prennent leur retraite.

Richard Titmus a fait remarquer qu'il y a en GrandeBretagne deux sortes de troisième âge: alors que certains retraités bénéficient d'une pension de leur employeur et du fruit de leurs épargnes, d'autres sont prestataires d'une pension de l'État (1955: 166). Les chercheurs britanniques Atkinson et Sutherland (1993 : 134) reprennent la même idée à propos des préretraités, présentant ce troisième âge à deux vitesses comme une illustration de la segmentation du marché du travail. Guillemard et Rein notent que ce dualisme ne s'est pas encore étendu au reste de l'Europe mais que ce sera bientôt chose faite si les divers États décident de réduire leurs dépenses sociales et de laisser au marché la liberté de prendre charge des travailleurs âgés (1993: 477). 


\section{DUALISME ECONOMIGUE ET RECHERCHE SUR LA RETRAITE}

À l'heure qu'il est, la recherche sur la théorie du dualisme économique et sur ses applications à la retraite reste assez mince. Ses pionniers ont cherché à montrer que les modèles structurels qu'elle propose fournissaient des explications plus satisfaisantes des comportements de retraite que les modèles basés sur les notions de capital humain et de réussite sociale; les plus récents travaux s'efforcent d'expliquer comment les secteurs influent sur le comportement de retraite et sur la structure de classe des retraités. Le courant qui domine actuellement ce champ de recherche s'intéresse davantage aux travailleurs plus jeunes (Furlong, 1990), à la distinction entre "bons" et "mauvais" emplois (Oi, 1990), à la mobilité professionnelle selon les secteurs (Wial, 1991), à la relation entre régionalisme et dualisme économique (Maier et Weiss, 1991) et au rôle du dualisme économique dans les pays en voie de développement (Nielsen, 1994). La plus grande partie de la recherche passée a tendu à appuyer les arguments de la théorie du dualisme économique suivant lesquels les conditions de travail sont meilleures au centre qu'à la périphérie (Mellow, 1983; Dickens et Lang, 1985), le rendement du capital humain tend à être plus élevé dans l'économie primaire, où les marchés du travail internes facilitent un développement plus poussé des qualifications (Dickens et Lang, 1985), et les salaires sont plus élevés dans le secteur central (Sakamoto et Chen, 1991).

Hendricks et McAllister (1983) ont été les premiers à prouver qu'un plus grand nombre de travailleurs du secteur central de l'économie que de travailleurs du secteur périphérique sont protégés par des régimes de retraite privés. Élaborant sur ce thème, Beck (1983), dans une étude sur la retraite non prévue, montre que les travailleurs masculins qui occupent des emplois à statut supérieur ou qui sont employés dans les industries du centre sont plus susceptibles de prendre leur retraite à l'âge prévu ou à un âge voisin, et que cet avantage semble lié à la protection que leur assurent un régime de retraite privé et la meilleure santé dont ils jouissent aux âges avancés. McDonald (1983), à l'aide de données américaines, confirme ces résultats, ajoutant que les travailleurs masculins du centre sont dans l'ensemble plus favorisés pour la plupart des caractéristiques socio-économiques et sont plus proches des comportements de retraite "normaux" correspondant à une carrière stable. En vérifiant l'influence respective du secteur économique et du capital humain sur le revenu de retraite, Leon 
(1985) constate que, dans le cas des Américains, c'est le modèle de type structurel (dualiste) qui offre la meilleure explication. Une étude visant à prédire l'effet du secteur économique sur la satisfaction éprouvêe après la retraite donne à penser que les considérations financières sont beaucoup importantes à cet égard pour les travailleurs de la périphérie, et la santé pour ceux du centre (Calasanti, 1988).

De leur côté, Mueller, Mutran et Boyle (1988) étudient les différences entre les secteurs au point de vue de la relation entre l'âge et le revenu gagné. S'il y a discrimination selon l'âge, elle devrait apparaître dans le centre de l'économie, où la rémunération de l'expérience devrait croître. Ils constatent très précisément qu'il y a discrimination salariale selon l'âge dans le secteur central et qu'elle se répand à mesure que les travailleurs vieillissent. Toutefois, Tigges (1988) montre de façon convaincante que les travailleurs du centre, jeunes et vieux, ont perdu du terrain au chapitre de la rémunération parce que depuis 1960 l'évolution de l'économie américaine ébranle les bases de leur pouvoir. La diminution des effectifs syndicaux, la transformation des catégories professionnelles et de l'industrie et les problèmes économiques du pays seraient à l'origine de ces changements. Les travailleurs du centre s'en ressentent parce que ce sont eux qui avaient le plus à perdre, ceux de la périphérie n'ayant rien à donner qui puisse fournir une marge de manœuvre aux gestionnaires en mal de changements organisationnels. Pour sa part, Nelson (1994) montre que le secteur d'activité économique - centre ou périphérie - n'est utile à la prédiction des avantages sociaux que dans la mesure où l'on sait s'il y a production d'un bien ou production d'un service : en désagrégeant le centre et la périphérie selon leurs éléments constitutifs, il constate que la localisation par grand secteur économique ne permet de prédire les avantages sociaux que pour l'industrie manufacturière, et non pour les services.

À partir de données du recensement canadien, McDonald et Wanner (1989) et McDonald (1989) constatent que la situation des retraités ne se présente pas de la même façon dans tous les secteurs de l'économie. Les hommes et les femmes qui travaillent dans les firmes du centre peuvent espérer prendre une retraite précoce et sont plus sûrs de jouir d'une pension privée. L'âge a un effet beaucoup plus marqué sur le nombre de semaines chômées dans le centre qu'à la périphérie, mais pour les hommes seulement. Une étude canadienne plus récente, visant à prédire la situation des retraités, confirme ces résul- 
tats mais précise, en ce qui concerne les travailleurs de la périphérie, qu'ils sont moins susceptibles d'avoir pris leur retraite parce qu'ils bénéficiaient d'une pension suffisante et moins susceptibles de cotiser à un régime de retraite lié à leur emploi et que, même s'ils se disent en moins bonne santé, ils travaillent plus longtemps que les travailleurs du centre. Dale et Bamford (1988), exploitant des données britanniques, constatent une érosion marquée des différences selon le sexe entre les hommes et les femmes qui continuent de travailler après avoir atteint l'âge normal de la retraite, ce qui donne à penser que l'âge de la retraite est plus flexible dans les secteurs de la périphérie. En Grande-Bretagne, l'étude empirique d'Atkinson et Sutherland (1993) soutient leur hypothèse de troisième âge à deux vitesses, du moins pour ce qui est de la retraite précoce; à l'instar de Myles et Teichroew (1991), ces auteurs mettent en cause les régimes de retraite publics et les régimes de retraite liés à l'emploi.

Si l'on a raison de considérer l'ordre économique comme un système où coexistent deux secteurs opposés et si ce dualisme marque le fonctionnement du système de protection de la retraite conformément aux hypothèses énoncées, on devrait constater des différences entre les ex-travailleurs du centre et ceux de la périphérie au chapitre des revenus et de divers éléments liés à la retraite. Les travailleurs qui ont fait carrière dans le centre devraient pouvoir prendre leur retraite plus tôt et jouir de meilleurs avantages sociaux et d'un régime de retraite privé, tandis que les retraités qui ont travaillé toute leur vie dans la périphérie seront moins susceptibles de prendre une retraite précoce et de bénéficier d'un régime de retraite privé; en outre, leur revenu de retraite devrait ètre inférieur à celui des ex-travailleurs du centre. Si les résultats confirment ces hypothèses, ils montreront à l'évidence quels retraités sont tributaires des régimes publics de retraite et permettront de dire si la structure économique existante et le régime de pensions dualiste qui l'accompagne favorisent la polarisation des revenus de retraite.

\section{LES RÉGIMES DE PENSIONS AU CANADA}

Un bref portrait du système canadien de protection de la retraite illustre la correspondance entre le caractère à la fois public et semi-privé de ce système et ce qu'ont vécu les retraités au cours de leur vie active dans un marché du travail segmenté. 
La composante publique du système comprend la Sécurité de la vieillesse, l'allocation au conjoint, le Régime de pensions du Canada (RPC) et le Régime de rentes du Québec (RRQ), le supplément de revenu garanti et un certain nombre de programmes provinciaux complémentaires ${ }^{4}$. Les prestations de sécurité de la vieillesse, instituées en 1951, sont versées à tous les Canadiens qui ont atteint l'âge de 65 ans, et elles sont ajustées tous les trois mois en fonction de l'indice des prix à la consommation ${ }^{5}$. Le gouvernement fédéral a mis fin à l'universalité de ce programme en 1989, en exigeant le remboursement de sommes versées à ce titre à des personnes dont le revenu était égal ou supérieur à 53000 dollars. L'allocation de conjoint, implantée en 1975, est versée aux personnes de 60 ans à 65 ans dont le conjoint bénéficie de prestations de sécurité de la vieillesse, s'il est démontré que leur revenu ne dépasse pas la limite permise. Elle est versée aussi aux veufs et veuves de 60 ans à 65 ans, sur vérification de leur revenu, mais non aux personnes divorcées, séparées ou célibataires ${ }^{6}$. Le Régime de pensions du Canada et le Régime de rentes du Québec, implantés en 1965, sont conçus pour verser des prestations ne dépassant pas $25 \%$ du revenu, revenu dont le plafond est égal au salaire industriel moyen. Les deux régimes prévoient des prestations pour le conjoint survivant et une rente d'invalidité, et sont financés par des cotisations obligatoires payées par les employés et les employeurs ${ }^{7}$. Le supplément de revenu garanti, qui a été institué à titre provisoire en 1967 et devait disparaître lorsque les deux régimes de pensions arriveraient à maturité, est devenu un programme permanent dont les prestations, sujettes à l'examen des revenus des bénéficiaires, peuvent être complètes ou partielles ${ }^{8}$. On peut s'attendre à ce que le revenu des retraités de la périphérie dépende plutôt des prestations de

4 Toutes les provinces canadiennes ont des programmes complémentaires, sauf quatre : le Nouveau-Brunswick, Terre-Neuve, l'Île-du-Prince-Édouard et le Québec.

5 Au cours de l'exercice 1993-1994, environ 15 milliards de dollars ont été versés en prestations à plus de trois millions de Canadiens (Ministère des Finances, 1994).

6 En 1994, les sommes distribuées à ce titre à 113000 prestataires se sont élevêes à quelque 400 millions de dollars (Statistique Canada, 1994).

7 On estime qu'en 1992,11,8 milliards de dollars ont été redistribués par l'entremise du Régime de pensions du Canada et du Régime de rentes du Québec (Canadian Institute of Actuaries, 1995).

8 En 1993-1994, les prestations versées à ce titre se sont élevées à environ 4,4 milliards de dollars, et $40,4 \%$ des bénéficiaires du programme de Sécurité de la vieillesse les ont touchées (Ministère des Finances, 1994). 
sécurité de la vieillesse et du supplément de revenu garanti que du RPC et du RRg, qui sont liés aux revenus d'emploi passés.

Au Canada, les programmes semi-privés comprennent les régimes de retraite liés à l'emploi, parfois appelés régimes de retraite agréés (RRA dans la suite du texte), et les régimes enregistrés d'épargne-retraite (REER). Les deux types de régimes sont organisés et gérés par le secteur privé mais sont subventionnés par des exemptions d'impôts associées aux cotisations versées et aux revenus accumulés, si bien qu'on les considère comme semi-privés. Le ministère canadien des Finances (1992) estime qu'en 1991 ces exemptions ont dépassé neuf milliards de dollars dans le cas des RRA et cinq milliards dans le cas des REER. Si l'on ajoute à ces avantages les régimes de participation différée aux bénéfices, on constate que le niveau de subvention par la taxation est de près de 15 milliards de dollars.

Les RRA sont plus courants dans le secteur public que dans le secteur privé. En 1986, près de $98 \%$ des employés du secteur public étaient couverts par un RRA, comparativement à seulement $32 \%$ des employés du secteur privé, et dans l'ensemble plus d'hommes (52\%) que de femmes ( $37 \%$ ) en bénéficiaient. La plupart des données canadiennes rêcentes montrent clairement que la participation à un REER est étroitement liée au revenu et augmente fortement à mesure que celui-ci s'élève (Brown, 1991; Weitz, 1992). On doit s'attendre à ce que les retraités qui travaillaient dans le centre (y compris le secteur étatique) tirent davantage profit des RRA; en outre, leurs revenus supérieurs devraient leur avoir permis d'accumuler plus d'épargne sous forme de REER.

La meilleure façon d'évaluer la performance globale du système public canadien de protection de la retraite consiste à la comparer à celle des autres pays du G7 et de l'Australie. Si l'on prend comme dénominateur le revenu d'une personne seule gagnant le salaire industriel moyen, le régime public canadien remplace $39 \%$ du revenu individuel antérieur à la retraite; les proportions correspondantes sont de $80 \%$ pour l'Italie, $50 \%$ pour la France et le Japon, $45 \%$ pour l'Allemagne, $41 \%$ pour les États-Unis et $39 \%$ pour la Grande-Bretagne. Le Canada ne devance que l'Australie, qui n'offre aux retraités qu'un programme dont les prestations sont liées à l'examen de leur revenu (Canadian Institute of Actuaries, 1993). Il importe de noter qu'ensemble, la sécurité de la vieillesse doublée du supplément de revenu garanti et le RPC ou le RRQ constituent $54,9 \%$ du revenu des femmes âgées seules, dont $47 \%$ vivent 
dans la pauvreté ${ }^{9}$. La dépendance des femmes à l'égard de l'État est donc particulièrement aiguë parce que leur cheminement de carrière est interrompu et moins long et qu'elles occupent les emplois moins bien rémunérés que l'on trouve habituellement dans la périphérie de l'économie. Il en résulte, sur toute la durée de leur vie, des salaires plus bas et une plus faible participation aux régimes de retraite semi-privés.

\section{MÉTHODOLOGIE}

Les données utilisées ici sont tirées de l'Enquête sur le vieillissement et l'autonomie (EVA dans la suite du texte), qui a été menée auprès d'un sous-échantillon de l'Enquête sur la population active composé de 20036 répondants de 45 ans et plus, et portait sur la période comprise entre septembre 1990 et août 1991. Il s'agissait de mesurer les facteurs qui contribuent à la qualité de vie et à l'autonomie des personnes âgées d'aujourd'hui et de demain (voir le manuel des usagers du fichier de microdonnêes de l'EVA) ${ }^{10}$. Les données ont été recueillies en septembre 1991 par des intervieweurs de l'Enquête sur la population active, au cours d'entrevues de 30 minutes menées sur place (environ $10 \%$ des entrevues) ou par téléphone. Le questionnaire portait sur les principales caractéristiques socio-démographiques des répondants, sur leur santé, sur leur situation sociale et économique et sur leur comportement face à la retraite. Outre ce contenu spécifique à l'EVA, un certain nombre de variables tirées de l'Enquête sur la population active, telles que l'industrie, la profession et la durée de

9 La pauvreté des femmes âgées est liêe à de nombreux facteurs, notamment aux caractéristiques de leur participation au marché du travail et à leur rôle familial. Dans tous les métiers et professions et dans tous les secteurs industriels, les femmes sont généralement confinées aux emplois les moins bien rémunérés et, à cause du mariage et des grossesses, leur carrière est à la fois sujette à des interruptions et plus brève que celle des hommes. Comme elles ont, en revanche, une espérance de vie plus grande que ces derniers, elles sont plus susceptibles de connaittre le veuvage et la pauvreté qui lui est associée.

10 L'Enquête sur le vieillissement et l'autonomie a été menée à travers tout le pays par Statistique Canada, en collaboration avec Santé nationale et Bien-être social Canada, le Secrétariat du troisième âge, Condition physique et Sport amateur, Consommation et Corporations Canada, la Société canadienne d'hypothèques et de logement, le ministère des Anciens Combattants, le Secrétariat d'État et Communications Canada. Les instruments d'enquête ont êté conçus avec le concours du Réseau canadien de recherche sur le vieillissement (Canadian Aging Research Network). 
l'emploi, ont été ajoutées au fichier de microdonnées de l'EVA. Celle-ci est basée sur un échantillon complexe, stratifié, comportant plusieurs étapes de sélection et des probabilités inégales de sélection des répondants. Une pondération ajustée a été utilisée dans les analyses principales pour tenir compte de ces probabilités de sélection inégales, mais cette pondération ne tenait pas compte de la stratification et des regroupements inhérents au concept d'échantillonnage. Le sous-échantillon analysé ici comprend seulement les personnes ayant déjà fait partie de la main-d'œuvre qui se sont dites retraitées depuis 1989 au plus tard $(\mathrm{N}=4827)^{11}$.

On sait qu'il existe de nombreuses définitions de la retraite, selon les disciplines et les tendances théoriques des chercheurs. Les études récentes qui traitent de ce problème concluent qu'il n'existe pas de définition optimale (Parnes et Less, 1985; Ekerdt et DeViney, 1990). Les critères "objectifs" de la retraite, tels que la diminution du temps de travail et des gains, produisent des découpages et des exclusions arbitraires et risquent toujours de coller de trop près aux préoccupations du chercheur qui les propose (Ekerdt et DeViney, 1990 : 216). Quant aux définitions subjectives (autodéfinitions), elles pèchent par excès de prudence et sont sujettes aux interprétations diverses des répondants (Gibson, 1987; Belgrave, 1988; Ekerdt et DeViney, 1990). Malgré ces inconvénients, il appert que les diverses définitions donnent lieu à des résultats assez similaires (Diamond et Hausman, 1984; McDonald et Wanner, 1984; Palmore et al., 1985; Ekerdt et DeViney, 1990). Quant à nous, nous nous fions aux déclarations des répondants sur leur situation de retraités ou de non-retraités. Ce choix a les inconvénients de l'autodéfinition, mais, suivant une analyse dont nous ne ferons pas état, les classifications en fonction des

11 Selon la plupart des définitions, la personne qui prend sa retraite se retire du marché du travail, ce qui implique qu'auparavant elle travaillait ou participait de façon soutenue à la main-d'œuvre. Une telle définition est inexacte dans la mesure où les ménagères, qui peuvent n'avoir jamais été sur le marché du travail, travaillaient et, on le présume, ont pris leur retraite. Ces analyses éliminent une partie des femmes et des hommes de l'échantillon qui tiennent maison, prennent soin de quelqu'un ou sont euxmêmes atteints d'une incapacité chronique. Les processus qui influencent la prise de retraite ne sont vraisemblablement pas du tout les mèmes pour ces personnes que pour les femmes qui sont sur le marché du travail (Szinovacz, Ekerdt et Vinick, 1992). De même, étant donné l'évolution des modes de participation des femmes à la main-d'œuvre, à l'avenir, les cohortes de femmes qui atteindront l'âge de la retraite auront accumulé une plus grande expérience du marché du travail. 
déclarations des répondants, du nombre de semaines travaillées et du versement d'une pension par le gouvernement se recoupent largement.

Le revenu personnel déclaré sert ici de variable dêpendante. Les répondants devaient estimer leur revenu brut de toutes sources pour 1990 en le classant dans l'une des catégories proposées (moins de 5000 dollars à 80000 dollars et plus). Nous avons recodé le revenu au point milieu de chacune des catégories de 5000 dollars afin de créer une variable continue. Bien que l'on fasse habituellement subir aux données sur les gains une transformation par le logarithme naturel afin de réduire la pente de la distribution, pour cette variable, la meilleure transformation était la fonction de la racine carrée. Nous avons opté pour le revenu personnel annuel parce que c'était l'une des deux variables disponibles dans notre série de données, l'autre étant le revenu du ménage. Ce dernier brouille le portrait, en particulier en ce qui concerne les femmes, dont le revenu est souvent lié à leur état matrimonial (O'Rand et Henretta, 1982). En outre, nous n'avions aucun moyen de savoir si une autre personne contribuait au revenu du ménage, et certaines pensions, tel le supplément de revenu garanti, sont réduites si elles sont versées à un couple.

Il existe plusieurs manières d'opérationnaliser la notion de segmentation : tout dépend de ce que l'on cherche. Les diverses études abordent le clivage centre-périphérie à l'échelle du travailleur, de la firme, de l'établissement ou du secteur industriel (Clairmont, Apostle et Kreckel, 1983). Ceux qui s'intéressent au secteur économique utilisent habituellement les données relatives au secteur industriel, tandis que ceux qui veulent étudier les marchés du travail retiennent les caractéristiques individuelles des travailleurs. Notre mesure de la segmentation économique, qui repose sur les industries, a été créee par Statistique Canada à partir des codes à trois chiffres et du système de classification élaborés par Tolbert, Horan et Beck (1980). On a critiqué l'opérationnalisation au niveau industriel parce qu'elle serait trop générale pour être significative (Krahn et Lowe, 1993); on a soutenu aussi qu'il est plus utile de distinguer entre le centre et la périphérie pour le secteur manufacturier que pour les services (Granovetter, 1984; Tigges, 1988; Nelson, 1994). Mais ces inquiétudes ne semblaient pas justifiées pour la base de données que nous exploitons ${ }^{12}$.

12 Afin de vérifier l'influence des niveaux d'emploi (autonome et subordonné) du secteur central, nous avons défini une variable col bleu-col blanc, mais 
Quatre séries de variables indépendantes mesurant les caractéristiques démographiques et divers aspects du travail, du revenu et de la retraite sont incluses dans nos analyses. Les variables socio-économiques représentant le capital humain des travailleurs et les caractéristiques de travail dont on sait qu'elles exercent ou qui devraient exercer une influence sur le revenu de retraite, de même que les circonstances de la transition vers la retraite qui ont un lien direct avec le revenu de retraite, et enfin les sources de revenu représentant la dualité du système de pensions sont comprises dans les modèles. L'âge à la retraite, la taille du ménage, les années de scolarité, le niveau de qualification professionnelle, le prestige professionnel, le taux de chômage provincial, les deux variables de revenu et les deux variables de propriété du domicile sont continues. Les autres variables sont binaires (dummy) (voir à ce propos les notes du tableau 2).

L'âge à la retraite est inclus dans le modèle parce qu'il a été clairement démontré que le moment de la retraite influence fortement la situation matérielle des retraités. Les retraités précoces ont habituellement travaillé dans l'économie du centre, où ils sont plus susceptibles d'avoir suivi un cheminement de carrière normal débouchant sur une retraite anticipée très confortable (McDonald et Wanner, 1990) ${ }^{13}$. Dans l'analyse, le lieu de naissance remplace l'origine ethnique ${ }^{14}$; on sait que celle-ci influence de façon différentielle l'accès aux ressources sociales et économiques, et conséquemment le revenu de retraite (Wanner et McDonald, 1986). L'état matrimonial et la taille du ménage sont des indicateurs du niveau de consommation, des ressources financières et du soutien social, et sont inclus parce que les hommes mariés tendent à prendre leur retraite à un âge plus tardif, tandis que les femmes mariées tendent à la prendre plus jeunes, fait qui devrait influencer leur

elle n'a eu aucun effet sur l'analyse. Nous avons également utilisé une variable pour les biens et services pour voir si les distinctions proposées par Nelson (1984) se vérifiaient ici, mais elle n'a pas eu d'effet non plus.

On inclut habituellement l'àge et le carré de l'àge dans les modèles d'estimation du revenu pour tenir compte de la possibilité d'une relation non linéaire entre l'âge et le revenu. Les diagnostics de la régression montrent que la relation est linéaire, probablement parce que la variable dépendante présente moins de variance du fait que nous l'avons recodée au point milieu (régression à la moyenne) et que notre analyse ne porte que sur une petite partie du cycle de vie.

14 L'EVA comprenait des questions sur l'origine ethnique des répondants, mais cette information a été éliminée pour des raisons de confidentialité. 
revenu (O'Rand et Henretta, 1982; McDonald et Wanner, 1984; Hayward et Grady, 1990). Comme le montant des pensions est souvent ajusté en fonction du fait que le couple compte un ou deux retraités, le statut de retraité ou de non-retraité du conjoint ou de la conjointe est inclus. L'instruction, comme l'origine ethnique, donne lieu à un accès différentiel aux ressources sociales et économiques, et elle est habituellement associée à un âge à la retraite plus tardif, surtout pour les femmes (McDonald et Wanner, 1984). Il a été prédit que le rendement économique de l'instruction serait plus élevé dans le centre. Le milieu géographique est inclus, en tant qu'indicateur des différences de composition des industries et des professions, puisqu'il est bien connu que les travailleurs âgés demeurent liés au marché du travail pendant de plus longues périodes dans le secteur agricole (Méthot, 1987; O'Neill, 1994) et que les caractéristiques du marché du travail local influent sur le revenu (Mueller et al., 1988). Le taux de chômage provincial est inclus pour la même raison, et parce que l'on sait qu'au Canada environ $13 \%$ des personnes qui prennent leur retraite s'y décident parce qu'elles sont en chômage. Le taux de chômage provincial freinerait aussi le retour au travail après la retraite (environ $21 \%$ des hommes et $11 \%$ des femmes de l'échantillon ont déclarê être retournés sur le marché de l'emploi).

Parmi les caractéristiques reliées au travail, la préparation de la retraite, la réserve de richesse constituée par un régime de retraite lié à l'emploi et le retour au travail après la retraite auraient tous des effets sur le revenu de retraite (Pesando et Gunderson, 1988). Le niveau de qualification professionnelle est basé sur la classification des professions de Pineo, Porter et McRoberts (1977); les scores vont de 23,46 (travailleurs non qualifiés) à 72 (professionnels). Les niveaux de qualification supérieurs sont habituellement rêmunérés à des taux plus élevés, ce qui devrait en fin de compte influencer le revenu de retraite. La durêe d'emploi est utilisée comme indicateur approximatif de la stabilité de la carrière de travail, facteur susceptible d'influencer le revenu provenant des rêgimes de retraite publics et privés. Le travail autonome, habituellement plus répandu dans la périphérie, est inclus dans les modèles ${ }^{15}$. Les variables binaires représentant les motifs de la retraite -

15 Étant donné la diversité des régimes de retraite auxquels les travailleurs autonomes peuvent adhérer, nous avons aussi fait une régression en les excluant, mais cela n'a entrainé aucune espèce de modification significative des résultats des analyses. 
maladie, soin d'un proche, revenu suffisant, retraite obligatoire ${ }^{16}$, programme de retraite anticipée, emploi non disponible et désir de cesser de travailler - font partie des modèles parce que le mode de passage à la retraite influencerait le revenu qui la suit. Tous ces indicateurs s'appliquent au dernier emploi du répondant.

Pour établir l'influence de chacune des sources de revenu, nous incluons les variables représentant les régimes de retraite publics et privés. Nous ne disposions que des variables binaires comme indicateurs du fait que les répondants reçoivent ou non une pension de retraite, publique ou semi-privée. La sécurité de la vieillesse, le RPC et le RRQ, le supplément de revenu garanti et les rentes d'invalidité prennent la valeur de 1 si les répondants ont déclaré toucher un revenu de ces sources, de 0 dans le cas contraire. L'inclusion d'une variable sécurité de la vieillesse-supplément de revenu garanti est quelque peu contestable car elle combine une prestation fixe versée à tous et une prestation ajustée au revenu qui est payée seulement à certaines personnes. Le fait de toucher ou non des revenus de placements, de REER et de RRA a également été inclus; ces indicateurs des régimes semi-privés ont été codês de la même manière que ci-dessus. La variable de revenu du ménage est représentée par la racine carrée du revenu du ménage pour l'année 1990. La propriété du domicile est utilisée comme variable substitut représentant les actifs, de même que la valeur de la maison, la dette hypothécaire et l'existence d'une dette supérieure à 5000 dollars.

L'utilisation d'une base de données secondaires nous pose quelques difficultés. Certaines variables clès ne sont tout simplement pas disponibles; ainsi, nous ne savons pas si telle ou telle personne a travaillé à temps plein ou à temps partiel pendant la plus grande partie de sa vie, ni si elle travaillait dans le secteur public ou parapublic ou dans le secteur privé. En outre, les données ne sont pas longitudinales et nous devons nous fier à la mémoire des répondants en ce qui concerne leurs conditions de travail et les circonstances de leur retraite. Néanmoins, nous avons suffisamment d'information pour faire une évaluation préliminaire de la relation entre les politiques de retraite, l'économie et la situation financière des retraités.

16 Toutes les provinces canadiennes prévoient la retraite obligatoire sauf le Guébec, le Manitoba et le Nouveau-Brunswick. 
Nous avons mené une analyse bivariée comparant les extravailleurs du centre avec ceux de la périphérie afin de faire ressortir les différences fondamentales entre les deux groupes de retraités. Parallèlement, les modèles d'estimation du revenu permettent de mettre en évidence l'apport des divers facteurs au revenu des retraités, selon les secteurs. Nous avons fait une régression à partir d'une série réduite de variables, et une autre plus complète tenant compte de l'interaction entre certaines variables et le secteur économique, et captant l'effet des diverses variables selon le secteur où travaillait le retraité. Un test de Chow (1960) a été utilisé pour estimer la différence entre les pentes de ces deux modèles et est indiqué par un test $F$ mesurant la différence entre le $\mathrm{R}$ carré de chacun d'eux, tandis que les tests $t$ fournissent des tests séparés pour chaque variable. Une procédure de régression par sommets (ridge regression) a servi à pallier le problème des intercorrélations entre les variables (Hoerl et Kennard, 1970; Afifi et Clark, 1990). Nous avons réalisé des analyses séparées pour les hommes et pour les femmes à cause du cumul des différences entre les sexes eu égard à l'expérience sur le marché du travail, situation attribuable surtout au fait que les décisions des femmes en ce qui a trait au travail et à la retraite sont beaucoup plus sensibles aux responsabilités familiales qu'elles assument tout au long du cycle de vie (Henretta, O'Rand et Chan, 1993).

\section{RÉSULTATS}

\section{Analyse bivariée}

Le tableau 1 présente les moyennes et les pourcentages obtenus pour les caractéristiques socio-économiques et pour les caractéristiques relatives au travail et au revenu des hommes et des femmes retraités du centre et de la périphérie. On constate qu'il existe des différences statistiquement significatives entre les secteurs pour un certain nombre de caractéristiques clés.

De façon générale, les hommes qui travaillaient dans le centre vivent en milieu urbain et sont nés au Canada, comme on pouvait s'y attendre. Ils sont plus instruits, sont salariés et tendent à prendre leur retraite plus tôt que les hommes de la périphérie. À vrai dire, leur âge à la retraite est identique à l'âge moyen national pour les hommes. Il paraît logique qu'ils aient occupé moins longtemps le dernier emploi qu'ils ont détenu 
avant de prendre leur retraite étant donné leur âge plus précoce au moment de cet événement ${ }^{17}$. En ce qui concerne les variables relatives au travail, les travailleurs masculins du centre sont plus susceptibles d'avoir une pension liée à l'emploi, et ils ont une probabilité plus élevée d'avoir pris leur retraite parce qu'ils avaient un revenu suffisant, que leur employeur leur offrait un programme de retraite anticipée et que, tout simplement, ils désiraient cesser de travailler. Tous ces facteurs concordent avec le cheminement de carrière ordonné et planifié qui serait propre au centre de l'économie.

Mème si les hommes du centre ont travaillé moins tout en ayant le même niveau de qualification que les hommes de la périphérie, ils ont un revenu de retraite plus important que ces derniers. Ils jouissaient d'un revenu personnel et d'un revenu du ménage significativement plus élevés (d'environ 4000 dollars dans chaque cas), possédaient une maison de plus grande valeur mais grevée d'une hypothèque plus considêrable, avaient plus souvent des dettes supérieures à 5000 dollars et assumaient financièrement la responsabilité de quelqu'un d'autre. Nonobstant ces dépenses plus élevées, ils ont pris leur retraite plus tôt. Dans l'ensemble, leur dépendance à l'égard des régimes de retraite semi-privés est plus grande, dans la mesure où ils sont plus susceptibles de retirer un revenu d'un RRA et d'un REER et considèrent leurs pensions de retraite comme leur principale source de revenu. Comme on pouvait s'y attendre, une plus forte proportion d'entre eux se sont dits très satisfaits de leur revenu de retraite actuel. Par contre, les hommes retraités de la périphérie sont plus susceptibles d'être d'origine étrangère, sont moins scolarisés, sont moins susceptibles de vivre dans les zones urbaines et beaucoup plus susceptibles d'être travailleurs autonomes, et ils ne se montrent pas aussi satisfaits de leur revenu de retraite. Tout cela donne à penser que les travailleurs masculins du centre jouissent d'un revenu supérieur après avoir passé moins de temps sur le marché du travail, et profitent à la fois des régimes de retraite publics et semi-privés, comme nous l'avions prévu. Les retraités de sexe masculin qui travaillaient dans la périphérie de l'économie ont peiné plus longtemps pour un résultat moindre sur le plan financier, et leur revenu de retraite dépend davantage du régime public.

17 Le nombre de cas étant faible, les pourcentages obtenus pour la durée de l'emploi doivent être interprétés avec prudence. 
TABLEAU 1 - Moyennes et pourcentages pour les hommes et les femmes qui ont pris leur retraite en 1990-1991, par grand secteur économique, selon les données de l'Enquête sur le vieillissement et l'autonomie, 1991

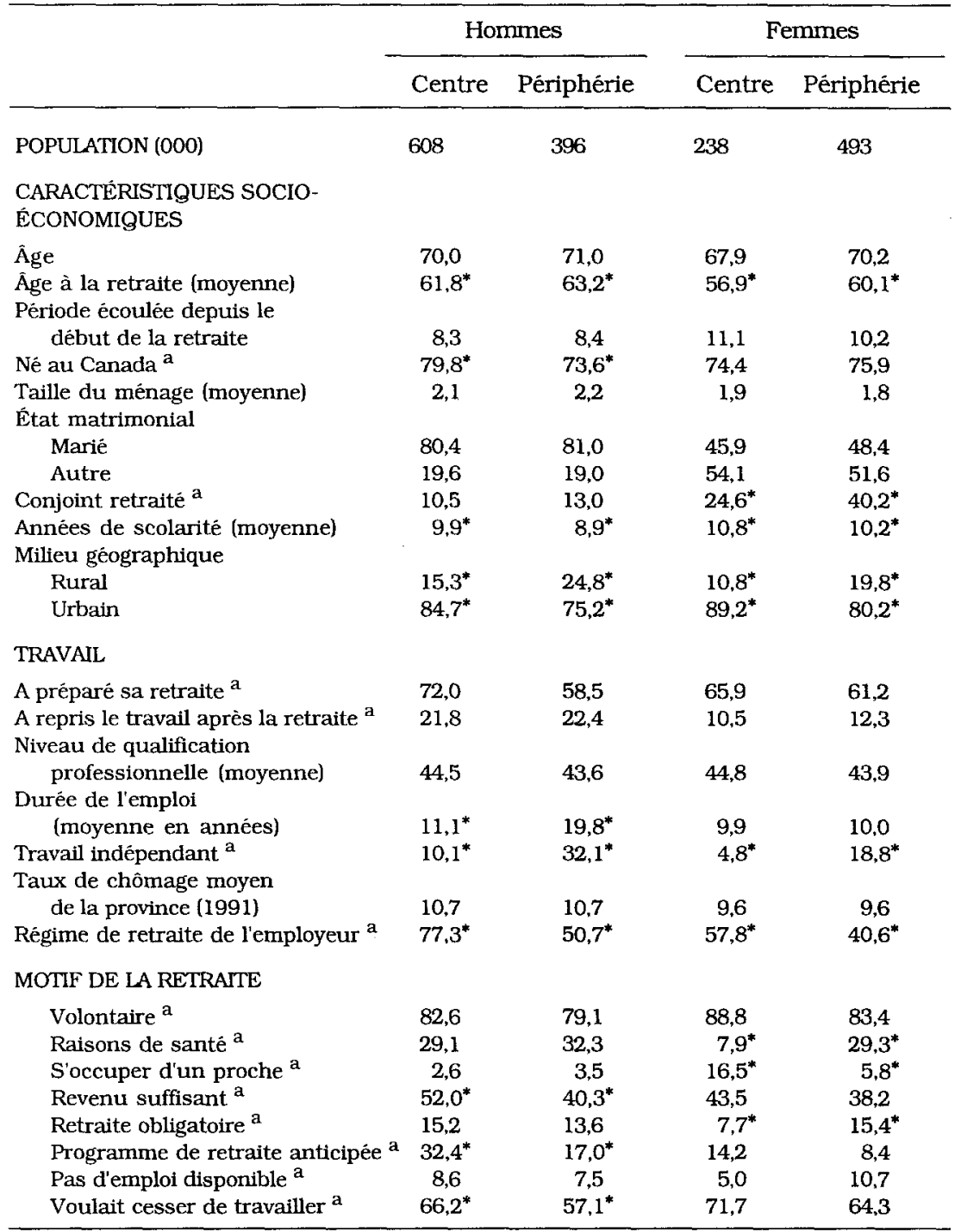

(suite du tableau page suivante)

En ce qui concerne les femmes, le tableau 1 montre clairement que celles qui travaillaient dans le centre sont favorisées. Elles sont plus susceptibles de vivre en milieu urbain et d'avoir pris leur retraite plus tôt que les femmes de la 
TABLEAU 1 - (suite)

\begin{tabular}{|c|c|c|c|c|}
\hline & \multicolumn{2}{|c|}{ Hommes } & \multicolumn{2}{|c|}{ Femmes } \\
\hline & Centre $P$ & Pêriphérie & Centre $\mathrm{F}$ & Périphérie \\
\hline \multicolumn{5}{|l|}{ REVENU } \\
\hline Revenu personnel (moyenne) & $27319.22^{*}$ & $22910,00^{*}$ & $18792,40^{*}$ & $15398,73^{*}$ \\
\hline Revenu pers. (moyenne corrigée) $b$ & $25022,24^{*}$ & $21010,10^{*}$ & $16460,17^{*}$ & $13541,60^{*}$ \\
\hline Revenu du mènage (moyenne) & $33398,24^{*}$ & $28442,37^{*}$ & $33474,82^{*}$ & $27476,78^{*}$ \\
\hline Rev. du ménage (moyenne corrigêe) & $30696.16^{*}$ & $26168,17^{*}$ & $30229.00^{*}$ & $24121,60^{*}$ \\
\hline Propriétaire de sa maison a & 80,3 & 81,7 & $72,2^{*}$ & $66,4^{*}$ \\
\hline Valeur de la maison & $112280,50^{*}$ & * $103632,30^{*}$ & $120113,70^{*}$ & $79823,20^{*}$ \\
\hline Dette hypothécaire & $31000.00^{*}$ & $14083.00^{*}$ & $14547,00^{*}$ & $6676,00^{*}$ \\
\hline Dettes dépassant $5000 \$^{a}$ & $4,3^{*}$ & $2,7^{*}$ & $3,6^{*}$ & $2,1^{*}$ \\
\hline \multicolumn{5}{|l|}{ Financièrement responsable } \\
\hline de quelqu'un & 18,1 & 16,9 & $9,5^{*}$ & $6,2^{*}$ \\
\hline Salaire & 5.9 & 7.5 & $4,7^{*}$ & $2,9^{*}$ \\
\hline Revenu de placements ${ }^{a}$ & 65,7 & 58,3 & $56,4^{*}$ & $48,9^{*}$ \\
\hline \multicolumn{5}{|l|}{ Transferts des gouvemements } \\
\hline $\mathrm{RPC}_{\mathrm{Cu}} \mathrm{RRg}^{\mathrm{a}}$ & 82,7 & 82,9 & 73,9 & 73,5 \\
\hline SV-SRG-AC a, c & $72,9^{*}$ & $80,8^{*}$ & $69.2^{*}$ & $75.8^{*}$ \\
\hline $\begin{array}{l}\text { Rente d'invalidité, assurance } \\
\text { sociale, etc. a }\end{array}$ & 9,9 & 9,7 & 8,1 & 7,9 \\
\hline \multicolumn{5}{|l|}{ Satisfaction à l'égard du revenu actuel } \\
\hline Très satisfait & $26,0^{*}$ & $22,4^{*}$ & $25,9^{*}$ & $20,9^{*}$ \\
\hline Satisfait & $63,1^{*}$ & $67,2^{*}$ & $61,0^{*}$ & $64,4^{*}$ \\
\hline Pas très satisfait & $9,5^{*}$ & $9,8^{*}$ & $12,8^{*}$ & $13,8^{*}$ \\
\hline Très insatisfait & $1,4^{*}$ & $0,6^{*}$ & $0,3^{*}$ & $0,9^{*}$ \\
\hline Revenu de RRA ${ }^{a}$ & $77,3^{*}$ & $50,7^{*}$ & $57,8^{*}$ & $40,6^{*}$ \\
\hline REER $^{a}$ & $55,3^{*}$ & $44,6^{*}$ & $47,2^{*}$ & $40,0^{*}$ \\
\hline \multicolumn{5}{|l|}{ Principale source de revenu } \\
\hline Aucune & $0,1^{*}$ & $0,1^{*}$ & $1,0^{*}$ & $1,0^{*}$ \\
\hline Travail & $2,1^{*}$ & $2,7^{*}$ & $0,6^{*}$ & $0,6^{*}$ \\
\hline Régime de retraite & $49,0^{*}$ & $29,7^{*}$ & $29,9^{*}$ & $19.8^{*}$ \\
\hline Pension du gouvernement & $35,3^{*}$ & $50,5^{*}$ & $48,4^{*}$ & $63,0^{*}$ \\
\hline Autres prestations gouv. & $2,6^{*}$ & $1,2^{*}$ & $1,1^{*}$ & $2,0^{*}$ \\
\hline Placements & $10,0^{*}$ & $14,9^{*}$ & $14,1^{*}$ & $12,2^{*}$ \\
\hline Autre (famille) & $0,1^{*}$ & $0,2^{*}$ & $1,1^{*}$ & $1,0^{*}$ \\
\hline Autre & $0,8^{*}$ & $0,5^{*}$ & $3,8^{*}$ & $0,4^{*}$ \\
\hline
\end{tabular}

* Les différences entre le centre et la périphérie sont significatives lorsque $\alpha \leq 0,05$.

a. Pourcentage de réponses affirmatives à la question. Les pourcentages ne totalisent pas 100 .

b. Moyenne corrigée : calculée après suppression des observations horschamp.

c. Sécurité de la vieillesse, supplément de revenu garanti, allocation de conjoint.

périphérie, elles ont un niveau d'instruction supérieur et leur conjoint est moins susceptible d'avoir déjà pris sa retraite. Toutefois, seulement deux caractéristiques reliées au travail distinguent les ex-travailleuses du centre de celles de la périphérie : le fait d'être salariées et le fait d'avoir participé à un 
régime de retraite lié à l'emploi. Les femmes de la périphérie sont en effet plus susceptibles d'être travailleuses autonomes, et partant, comme on pouvait s'y attendre, moins susceptibles de bénéficier d'un régime de retraite lié à l'emploi.

Les femmes du centre ont pris leur retraite pour des raisons qui sont plus liées à leur situation familiale qu'à leur situation de travail. Elles sont plus susceptibles d'avoir ainsi voulu se consacrer à un membre de leur famille. Une autre analyse reposant sur la même base de données a montré que l'âge des femmes à la retraite était de trois ans inférieur si elles avaient quitté leur emploi pour prendre soin de quelqu'un et que ce sont les femmes mariées de statut socio-économique supérieur et jouissant d'un revenu personnel et d'un revenu du ménage plus élevés qui posaient ce geste. Les résultats présentés ici vont dans le même sens. Les femmes de la périphérie ne quittent pas leur emploi pour prendre soin de quelqu'un, sans doute parce qu'elles n'en ont pas les moyens. Elles le font parce qu'elles sont susceptibles d'être malades et soumises à la retraite obligatoire. Il se peut que leur santé soit compromise par un travail difficile et pénible, mais la retraite obligatoire peut contrarier leur désir de reporter leur sortie du marché du travail afin d'avoir droit à la totalité de la pension versée par les régimes publics ou semi-privés.

Les femmes du centre sont en bien meilleure posture que les femmes de la périphérie pour la plupart des variables économiques. Comme les hommes de leur secteur, elles bénéficient d'un revenu personnel et d'un revenu du ménage supérieurs et sont plus susceptibles d'être propriétaires de leur maison; de même, cette maison a plus de valeur et son hypothèque est plus importante. Comme les hommes encore, elles sont plus susceptibles d'avoir des dettes dépassant 5000 dollars et d'être financièrement responsables de quelqu'un d'autre. Elles sont aussi plus susceptibles de recevoir un salaire, ce qui laisse croire qu'au moment de l'enquête elles conservaient un lien avec le marché du travail. En ce qui concerne les rêgimes de retraite semiprivés, les femmes du centre sont plus susceptibles d'avoir cotisé à un REER, d'avoir des revenus de placements et de toucher une pension privée. Elles sont moins susceptibles d'avoir déclaré que les transferts gouvernementaux constituent leur principale source de revenu et sont, dans l'ensemble, plus satisfaites de leur revenu que les femmes de la périphérie. Ces dernières vivent en fait sur le seuil de la pauvreté, ou presque, peu importe l'indicateur de pauvreté utilisé. 
Ainsi que nous l'avons noté plus haut en parlant des politiques de retraite du Canada, quel que soit le secteur où se trouvent les femmes, elles dépendent davantage des régimes publics de retraite. Comme le montre le tableau $1,48,4 \%$ des femmes qui travaillaient dans le centre ont déclaré que leur principale source de revenu était une pension du gouvernement, comparativement à $35,3 \%$ des hommes du centre, tandis que $63 \%$ des femmes et $50,5 \%$ des hommes qui travaillaient dans le secteur périphérique ont fait de même. Ce résultat, qui ne surprend pas, est très probablement attribuable au cheminement de carrière différent des femmes et en conséquence aux avantages moins intéressants, pour le moins, que leur procure leur travail (Ghalam, 1994). Il est fermement établi qu'au Canada les femmes sont concentrées dans des emplois qui donnent des salaires et des pensions plus faibles (Hughes et Lowe, 1994; Ghalam, 1994); on sait également qu'elles suivent une carrière en dents de scie et que celles qui occupent des postes équivalents à ceux des hommes gagnent $66 \%$ du salaire de ces derniers (Ghalam, 1994). Le secteur économique ne fait en somme qu'aggraver la situation des femmes, en accentuant le clivage entre les pauvres.

À ce stade, il paraît évident que le revenu de retraite tant des hommes que des femmes diffère en fonction du secteur économique où ils ont fait carrière et que les politiques de retraite semblent épouser la segmentation par secteur. Les hommes et les femmes du centre de l'économie sont tributaires à la fois des régimes de retraite publics et semi-privés, tandis que le revenu des retraités de la périphérie dépend davantage des régimes publics. Pour établir adéquatement l'apport de chaque source de revenu de retraite, nous avons aussi estimé des modèles multivariés pour les hommes et pour les femmes, par secteur.

\section{Analyse multivariée}

Le tableau 2 présente une série de régressions dans lesquelles le revenu personnel est la variable dépendante. $\mathrm{Ce}$ modèle comprend la plupart des variables socio-économiques, de travail, de retraite et de revenu ${ }^{18}$. Celui où sont ajoutées des variables tenant compte de l'interaction avec le secteur écono-

18 Nous avons retiré de l'analyse un certain nombre de variables, telles que la retraite du conjoint (en raison de sa collinéarité avec l'état matrimonial), la durée de l'emploi (dont la présence réduisait le nombre de cas) et plusieurs sources de revenu (interreliées). 
TABLEAU 2 -Régressions par sommets du revenu personnel avec les caractéristiques socio-économiques, professionnelles et de travail, hommes et femmes retraités, selon les données de l'Enquête sur le vieillissement et l'autonomie, $1991^{\text {a }}$

\begin{tabular}{|c|c|c|c|c|}
\hline \multirow{2}{*}{$\begin{array}{l}\text { Variables } \\
\text { prédéterminées }\end{array}$} & \multicolumn{2}{|c|}{ Hommes } & \multicolumn{2}{|c|}{ Femmes } \\
\hline & Centre & Périphérie & Centre & Périphérie \\
\hline \multicolumn{5}{|l|}{$\begin{array}{l}\text { CARACTÉRISTIQUES } \\
\text { SOCIO-ÉCONOMIQUES }\end{array}$} \\
\hline Né au Canada ${ }^{b}$ & $\begin{array}{l}2,01 \\
(0,017)\end{array}$ & $\begin{array}{l}-0,59 \\
(-0,001)\end{array}$ & $\begin{array}{l}3,25 \\
(0,028)\end{array}$ & $\begin{array}{l}-0,97 \\
(-0,011)\end{array}$ \\
\hline État matrimonial ${ }^{b}$ & $\begin{array}{l}-2,92 \dagger \\
(-0,025)\end{array}$ & $\begin{array}{c}1,17 \dagger \\
(0,016)\end{array}$ & $\begin{array}{l}-4,65^{*} \\
(-0,052)\end{array}$ & $\begin{array}{l}-7,10^{*} \\
(-0,093)\end{array}$ \\
\hline Taille du mênage & $\begin{array}{l}-0,89 \dagger \\
(-0,016)\end{array}$ & $\begin{array}{c}0,15 \dagger \\
(0,003)\end{array}$ & $\begin{array}{l}0,36 \\
(0,009)\end{array}$ & $\begin{array}{l}-1,78^{*} \\
(-0,042)\end{array}$ \\
\hline Années de scolarité & $\begin{array}{r}1,22^{*} \dagger \\
(0,145)\end{array}$ & $\begin{array}{l}1,12^{*} \dagger \\
(0,117)\end{array}$ & $\begin{array}{r}1,08^{* \dagger} \\
(0,094)\end{array}$ & $\begin{array}{l}0,826^{*} \dagger \\
(0,088)\end{array}$ \\
\hline Milieu géographique ${ }^{b}$ & $\begin{array}{l}-7,82^{* \dagger} \dagger \\
(-0,063)\end{array}$ & $\begin{array}{r}-4,49^{*} \dagger \\
(-0,045)\end{array}$ & $\begin{array}{l}-7,94^{*}+ \\
(-0,060)\end{array}$ & $\begin{array}{c}-0,41 \dagger \\
(-0,004)\end{array}$ \\
\hline \multicolumn{5}{|l|}{ TRAVAIL } \\
\hline Statut socio-économique ${ }^{c}$ & $\begin{array}{r}0,53^{*} \dagger \\
(0,145)\end{array}$ & $\begin{array}{r}0,45^{*} \dagger \\
(0,136)\end{array}$ & $\begin{array}{r}0,58^{* \dagger} \\
(0,162)\end{array}$ & $\begin{array}{r}0,41^{*} \dagger \\
(0,132)\end{array}$ \\
\hline Travail indépendant ${ }^{b}$ & $\begin{array}{l}-3,39 \\
(-0,023)\end{array}$ & $\begin{array}{l}0,19 \\
(0,002)\end{array}$ & $\begin{array}{l}-4,98 \\
(-0,021)\end{array}$ & $\begin{array}{l}0,64 \\
(0,006)\end{array}$ \\
\hline Âge à la retraite & $\begin{array}{l}-0,05 \dagger \\
(-0,004)\end{array}$ & $\begin{array}{r}-0,27^{*} t \\
(-0,032)\end{array}$ & $\begin{array}{c}0,02 \\
(0,003)\end{array}$ & $\begin{array}{l}-0,02 \\
(-0,004)\end{array}$ \\
\hline A repris le travail après la retraite ${ }^{b}$ & $\begin{array}{c}0,41 \\
(0,003)\end{array}$ & $\begin{array}{c}4,66^{*} \\
(0,047)\end{array}$ & $\begin{array}{r}8,22^{*} \dagger \\
(0,063)\end{array}$ & $\begin{array}{c}3,30 \dagger \\
(0,030)\end{array}$ \\
\hline MOTIF DE LA RETRAITE & & & & \\
\hline Maladie $^{b}$ & $\begin{array}{l}-7,15^{*} \dagger \\
(-0,068)\end{array}$ & $\begin{array}{l}-3,65^{*} \dagger \\
(-0,041)\end{array}$ & $\begin{array}{l}-2,78 \\
(-0,029)\end{array}$ & $\begin{array}{l}-6,13^{*} \\
(-0,076)\end{array}$ \\
\hline S'occuper d'un proche ${ }^{b}$ & $\begin{array}{l}-0,75 \\
(-0,003)\end{array}$ & $\begin{array}{c}4,74 \\
(0,022)\end{array}$ & $\begin{array}{l}-7,57^{* \dagger} \\
(-0,052)\end{array}$ & $\begin{array}{l}-2,50 \dagger \\
(-0,022)\end{array}$ \\
\hline Retraite obligatoire ${ }^{b}$ & $\begin{array}{l}-2,59 \dagger \\
(-0,025)\end{array}$ & $\begin{array}{r}3.73^{*} \dagger \\
(0,035)\end{array}$ & $\begin{array}{l}0,61 \\
(0,005)\end{array}$ & $\begin{array}{l}-1,12 \\
(-0,011)\end{array}$ \\
\hline $\begin{array}{l}\text { Programme de retraite anticipée } \\
\text { de l'employeur } b\end{array}$ & $\begin{array}{c}5,44^{* \dagger} \\
(0.048)\end{array}$ & $\begin{array}{r}9,04^{* \dagger} \\
(0,059)\end{array}$ & $\begin{array}{c}4,79 \\
(0,025)\end{array}$ & $\begin{array}{c}6,31^{*} \\
(0,038)\end{array}$ \\
\hline Pas d'emploi disponible ${ }^{b}$ & $\begin{array}{l}-6,48 \\
(-0,026)\end{array}$ & $\begin{array}{l}-9,09^{*} \\
(-0,054)\end{array}$ & $\begin{array}{l}-1,23 \\
(-0,007)\end{array}$ & $\begin{array}{l}-5,61^{*} \\
(-0,037)\end{array}$ \\
\hline
\end{tabular}

(suite du tableau page suivante)

mique n'est pas reproduit ici, bien que la prise en compte de ces effets ait été significative tant pour les hommes que pour les femmes. Dans le tableau, les croix identifient les variables qui n'exercent pas la mème influence dans les deux secteurs.

La justesse des modèles, indiquée par le carré de la corrélation multiple, est relativement satisfaisant, puisque le pourcentage de variance expliquée atteint 41 pour les hommes du centre et 38 pour ceux de la périphérie, et s'êlève à 34 pour les 
TABLEAU 2 - (suite)

\begin{tabular}{|c|c|c|c|c|}
\hline \multirow{2}{*}{$\begin{array}{l}\text { Variables } \\
\text { prédéterminées }\end{array}$} & \multicolumn{2}{|c|}{ Hommes } & \multicolumn{2}{|c|}{ Femmes } \\
\hline & Centre & Périphérie & Centre & Périphérie \\
\hline \multicolumn{5}{|l|}{ REVENU } \\
\hline Propriétaire de sa maison ${ }^{b}$ & $\begin{array}{r}6,19^{*} \dagger \\
(0,056)\end{array}$ & $\begin{array}{l}3,10^{\dagger} \\
(0,029)\end{array}$ & $\begin{array}{l}-1,49 \\
(-0,017)\end{array}$ & $\begin{array}{l}1,91 \\
(0,025)\end{array}$ \\
\hline Revenu d'un RRA ${ }^{b}$ & $\begin{array}{r}13,72^{* \dagger} \\
(0,126)\end{array}$ & $\begin{array}{l}12,31^{* \dagger} \\
(0,146)\end{array}$ & $\begin{array}{l}6,85^{*} \\
(0,076)\end{array}$ & $\begin{array}{l}11,63^{*} \\
(0,151)\end{array}$ \\
\hline Revenu de placements ${ }^{b}$ & $\begin{array}{l}11,96^{*} \dagger \\
(0,127)\end{array}$ & $\begin{array}{l}10,06^{*} \dagger \\
0,117)\end{array}$ & $\begin{array}{l}13,88^{*} \dagger \\
(0,155)\end{array}$ & $\begin{array}{r}8,73^{*} \dagger \\
(0,117)\end{array}$ \\
\hline Revenu d'un REER ${ }^{b}$ & $\begin{array}{r}12,02 * \dagger \\
(0,131)\end{array}$ & $\begin{array}{c}8,66^{*} \dagger \\
(0,101)\end{array}$ & $\begin{array}{l}10.46^{*} \dagger \\
(0,121)\end{array}$ & $\begin{array}{r}5,53^{*}+ \\
(0,071)\end{array}$ \\
\hline Revenu du RPC ou du RRg ${ }^{b}$ & $\begin{array}{l}6,98 \\
(0,026)\end{array}$ & $\begin{array}{l}6,38^{*} \\
(0,033)\end{array}$ & $\begin{array}{l}9,67^{*} \\
(0,055)\end{array}$ & $\begin{array}{c}1,45 \\
(0,012)\end{array}$ \\
\hline Revenu de SV-SRG-AC ${ }^{\text {b, d }}$ & $\begin{array}{c}-5,56^{*} \dagger \\
(-0,045)\end{array}$ & $\begin{array}{r}-6,69^{*} \dagger \\
(-0,044)\end{array}$ & $\begin{array}{c}4,17 \\
(0,031)\end{array}$ & $\begin{array}{c}0,12 \\
(0,001)\end{array}$ \\
\hline Revenu de transferts gouvern. ${ }^{b}$ & $\begin{array}{r}7,39^{*} \dagger \\
(0,048)\end{array}$ & $\begin{array}{c}2,36 \dagger \\
(0,016)\end{array}$ & $\begin{array}{r}11,43^{*} \dagger \\
(0,083)\end{array}$ & $\begin{array}{c}3,99 \dagger \\
(0,025)\end{array}$ \\
\hline $\begin{array}{l}\text { POINT D'INTERSECTION } \\
\mathbf{R}^{2} \\
\mathrm{R}^{2} \text { ajusté }\end{array}$ & $\begin{array}{l}96,43 \\
0,4247 \\
0,4105\end{array}$ & $\begin{array}{r}111,43 \\
0,4054 \\
0,3847\end{array}$ & $\begin{array}{c}65,75 \\
0,3995 \\
0,3479\end{array}$ & $\begin{array}{r}88,03 \\
0,3476 \\
0,3274\end{array}$ \\
\hline Nombre de cas & 869 & 624 & 266 & 699 \\
\hline
\end{tabular}

a. Tous les coefficients sont des estimations non standardisées; les coefficients standardisés sont entre parenthèses.

* Dans les modèles des effets de premier ordre, les coefficients sont significativement différents de 0 lorsque $\alpha \leq 0,05$.

$\dagger$ Les coefficients correspondants du centre et de la périphérie sont significativement différents lorsque $\alpha \leq 0,05$, d'après des tests de Chow (non présentés).

b. Définition des variables binaires: la variable vaut 1 si le répondant $(R)$ est : né au Canada; a pris sa retraite pour s'occuper d'un proche; a cotisé à un REER; est marié; a été obligé de prendre sa retraite (retraite obligatoire); touche un revenu du RPC ou du RRQ; vit en milieu rural; a bénéficié d'un programme de retraite anticipée; reçoit une pension de sécurité de la vieillesse, une rente d'invalidité ou de l'aide sociale; est travailleur autonome; a pris sa retraite parce qu'il ne trouvait pas de travail; est retourné au travail après avoir pris sa retraite; touche une pension d'un régime de retraite privé; reçoit de l'assurance-chômage; a pris sa retraite pour raisons de santé; a des revenus de placements.

c. Selon la classification socio-économique des professions de Pineo, Porter et McRoberts (1977).

d. Sécurité de la vieillesse, supplément de revenu garanti, allocation de conjoint.

femmes du centre et à 32 pour celles de la périphérie. Il est évident que les pourcentages sont inférieurs pour les femmes parce que les modèles ne tiennent pas compte du fait que leur dernier emploi ait été à temps plein ou à temps partiel et qu'ils ne captent pas les mouvements d'entrée et de sortie qui 
ponctuent leurs rapports avec le marché du travail tout au long de leur vie active ${ }^{19}$.

En examinant les résultats du modèle d'estimation des effets de premier ordre, on constate que, pour les hommes qui travaillaient dans le centre, les variables clés, classées par ordre d'importance en fonction des coefficients standardisés ${ }^{20}$, sont le statut socio-économique, les revenus provenant d'un REER, les revenus de placements, les revenus de pensions et le niveau d'instruction. Les résultats associés à la première constellation de variables concordent avec ceux de l'analyse bivariée, qui faisaient ressortir l'apport des régimes de retraite semi-privés au revenu de retraite. C'est surtout le fait d'avoir un REER, des placements et un régime de retraite privé qui influence le revenu des retraités du secteur central de l'économie. Ce revenu est complété par les régimes publics, car le RPC et le RRQ ainsi que les autres paiements de transfert gouvernementaux, tels que les rentes d'invalidité et l'aide sociale, exercent un effet notable, quoique moindre. La forte influence des autres paiements de transfert gouvernementaux sur le revenu des hommes du centre résulte probablement du rôle des rentes d'invalidité dans le processus de transition vers la retraite; ce résultat, qui concorde avec l'âge à la retraite plus précoce de ces hommes, correspond à un phénomène abondamment observé en Europe (Guillemard, 1991). La valeur négative obtenue pour la sécurité de la vieillesse et le supplément de revenu garanti exprime probablement le fait que seules les personnes à très faible revenu touchent le second.

La propriété du domicile, utilisée comme mesure approximative des actifs, est associée à un revenu de retraite plus élevé, comme on pouvait s'y attendre. Le fait d'avoir bénéficié d'un programme de retraite anticipée de l'employeur a un effet positif, susceptible de traduire la générosité de ces dispositifs destinés à faire sortir les hauts salariés de la main-d'œuvre (Myles et Street, 1995); l'effet positif des deux variables de "réussite" (statut socio-économique et niveau d'instruction) est bien connu dans le champ de la recherche sur la segmentation économique (Sakamoto et Chen, 1991). Les coefficients négatifs

19 À l'aide des données de l'Enquête sur l'activité, McDonald et Wanner (1992) ont montré que parmi les femmes qui ont un emploi à temps partiel, $34,5 \%$ travaillent dans le centre et $65,5 \%$ dans la périphérie.

20 Le coefficient de régression standardisé est une valeur statistique permettant de comparer l'importance relative de diverses variables dans une analyse de régression multiple (Vogt, 1993). 
obtenus pour la santé et le fait de vivre en milieu rural agissent dans le sens attendu. La maladie est un événement imprévu qui, ultimement, devrait diminuer le revenu de retraite, en contraignant le travailleur à une cessation d'activité précoce à laquelle il ne s'est pas préparé. On sait par ailleurs que, dans les zones rurales, le salaire et le revenu sont moins élevés (Conseil économique du Canada, 1991).

Le portrait obtenu en ce qui concerne le revenu est le même pour les hommes de la périphérie que pour ceux du centre. Un examen des coefficients standardisés indique que le revenu d'un régime de retraite privé, le statut socio-économique, le niveau d'instruction, les revenus de placements et un REER produisent la plus forte augmentation du revenu annuel de retraite. Le RPC et le RRQ et la sécurité du revenu couplée avec le supplément de revenu garanti sont les seuls programmes publics qui influencent le revenu des hommes de la périphérie et, relativement aux autres coefficients, ils n'exercent pas un effet aussi marqué sur le revenu de retraite. La sécurité de la vieillesse présente une forte corrélation négative avec le revenu parce que, comme on l'a vu au tableau 1, le revenu est moins élevé à la périphérie que dans le centre.

Contrairement à ce qui se passe pour les retraités du centre, dans le cas des retraités de la périphérie, le retour au travail est fortement corrélé avec une augmentation du revenu de retraite; encore une fois, il s'agit peut-être d'une caractéristique imputable au revenu plus faible auquel ils ont droit au début de leur retraite, et c'est sans doute ce fait qui les incite à retourner au travail. On constate, sans surprise, que la retraite obligatoire est associée à une augmentation du revenu des retraités de la périphérie; l'explication présumée est qu'elle a pour effet de les retenir sur le marché du travail pendant une période plus longue. La seule autre différence observée dans le modèle en ce qui concerne la périphérie a trait à la retraite causée par l'absence de travail, fortement corrélée à une diminution du revenu pour les retraités de ce secteur, qui ont évidemment des moyens de subsistance moindres, surtout s'ils perdent leur emploi.

Un test de Chow (1960) appliqué aux différences entre les pentes obtenues pour les hommes du centre et ceux de la périphérie indique que les deux modèles sont significativement différents. On remarque les principales différences dans les coefficients associés à l'âge à la retraite, à l'instruction, à l'état matrimonial, au statut socio-économique, à la taille du ménage et au milieu géographique, de même qu'au fait de 
recevoir une pension, d'avoir des revenus de placements, d'avoir cotisé à un REER, de recevoir une pension de sécurité de la vieillesse et un supplément de revenu garanti et d'autres transferts gouvernementaux, et enfin au fait d'être retraité pour raisons de santé, d'avoir bénéficié d'un programme de retraite anticipée de l'employeur et d'avoir étê soumis à la retraite obligatoire. Lorsque l'on tient compte de l'interaction avec le secteur économique, ce sont les variables associées au revenu qui présentent le plus d'effets.

Nos résultats soutiennent assez éloquemment l'hypothèse d'une polarisation du revenu de retraite épousant les divisions associées à la segmentation de l'économie. Les retraités des industries du centre sont beaucoup plus avantagés pour la quasi-totalité des indicateurs de revenu et de réussite. Les extravailleurs de la périphérie sont tributaires des mêmes sources de revenu que ceux du centre, mais le revenu qu'ils tirent de toutes ces sources n'est pas aussi fortement corrélé avec l'augmentation de leur revenu de retraite, même si l'on tient compte des paiements de transfert gouvernementaux. Ainsi, le fait de cotiser à un REER n'influence pas autant le revenu de retraite des travailleurs de la périphérie que celui des travailleurs du centre. L'effet d'un revenu annuel provenant d'une pension privée est également moindre pour les travailleurs de la périphérie, même quand ils prennent une retraite plus tardive. Les revenus de placements sont associés à une plus forte augmentation de revenu pour les ex-employés du centre que pour ceux de la périphérie, tandis que la propriété du domicile (indicateur approximatif des actifs) est plus fortement corrélée avec un revenu supérieur pour les retraités du centre, mais n'a pas d'effet pour les retraités de la périphérie.

Tous les autres termes qui tiennent compte de l'interaction avec le secteur présentent une concordance avec les différences de conditions de travail observêes entre le centre et la périphérie. Parmi les variables socio-démographiques, l'état matrimonial, qui sert ici à mesurer les ressources économiques et les dépenses, aurait un impact plus net dans le centre parce que les hommes y prennent leur retraite plus tôt et assument la responsabilité financière d'un plus grand nombre de personnes, sont plus endettés et assument de plus lourdes hypothèques. Le fait de travailler en milieu rural ne semble guère modifier le revenu déjà faible des hommes de la périphérie mais réduit le revenu des hommes des industries du centre, qui devraient normalement jouir d'un salaire plus élevé dans 
les régions urbaines. L'âge à la retraite exerce une influence négative plus forte à la périphérie que dans le centre, où les travailleurs qui prennent une retraite anticipée jouissent normalement de revenus de retraite substantiels. L'effet significatif du secteur que l'on obtient pour le statut socioéconomique montre que, lorsque les travailleurs des deux groupes se situent au même niveau eu égard à cette variable, ceux du centre touchent une rémunération plus élevée que ceux de la périphérie; c'est un signe évident que le capital humain ne reçoit pas la même rémunération dans les deux secteurs (Sakamoto et Chen, 1991). L'effet de la maladie en tant que motif de la retraite est plus fort dans le centre, probablement parce que les rentes d'invalidité y sont plus accessibles aux travailleurs. L'effet négatif de la retraite obligatoire sur les revenus dans le centre est contraire aux attentes, mais il peut être attribuable au fait que les programmes de retraite anticipée, habituellement destinés aux hauts salariés, en ont incité plusieurs à se retirer de ce secteur. D'un point de vue relatif, les travailleurs du centre qui travaillent jusqu'à l'âge de la retraite obligatoire sont les moins bien protégés financièrement. Les travailleurs de la périphérie sont plus susceptibles de rester sur le marché du travail afin de retirer leurs pleines prestations de retraite plutôt que des prestations diminuées, car leur revenu de base est inférieur. De même, l'impact d'un programme de retraite anticipée de l'employeur est plus marqué pour les ex-travailleurs de la périphérie.

Dans l'ensemble, il apparaît que les retraités masculins de la périphérie se trouvent dans une situation financière plus précaire, principalement parce que leur revenu de retraite dépend davantage des régimes publics (tableau 1) et qu'aucune des sources publiques de revenu, sauf le RPC et le RRQ, n'est aussi avantageuse pour eux que ce que reçoivent les hommes qui travaillaient sur le marché du travail primaire. Les sources de revenu semi-privées sont associées à de plus fortes augmentations du revenu de retraite que les sources de revenus publiques, mais ces augmentations restent inférieures à celles dont jouissent les travailleurs du centre, qui sont les véritables bénéficiaires du système dualiste de pensions de retraite.

Le portrait qui se dégage du modèle d'estimation des effets de premier ordre pour les femmes du centre est le même que pour les hommes des deux secteurs de l'économie. Le fait qu'elles aient un revenu de retraite plus élevé est associé aux régimes de retraite semi-privés, c'est-à-dire au fait qu'elles 
aient des placements, un REER et, à un degré moindre, un RRA. Le RPC et le RRQ, ainsi que les autres paiements de transfert gouvernementaux, sans faire augmenter le revenu de retraite des femmes de façon aussi marquée que les sources semiprivées, l'influencent fortement. Les autres variables significatives fonctionnent à peu près de la même manière que pour les hommes, sauf le retour au travail postérieur à la retraite et le soin d'un proche en tant que motif de la retraite. Si les retraitées du centre reprennent le travail, elles augmentent leur revenu de façon radicale, ce qui ne saurait surprendre, car elles ont un statut socio-économique plus élevê et plus d'années de scolarité que les autres répondants. La retraite précoce est aussi de nature à faciliter leur retour au travail. Mais en prenant leur retraite pour s'occuper d'un membre de leur famille, elles subissent une forte perte de revenu, d'autant plus qu'elles sont susceptibles d'avoir un revenu de retraite satisfaisant au départ.

En comparaison, le portrait obtenu pour les retraitées de la périphérie, dont $19,8 \%$ ont déclaré que les régimes de retraite privés étaient leur principale source de revenu (voir le tableau 1), illustre que le revenu de ces régimes est fortement corrélé avec une augmentation du revenu de retraite. L'absence de corrélation significative entre la jouissance d'un revenu de source gouvernementale et l'augmentation du revenu de retraite témoigne de la situation précaire des femmes du secteur périphérique. Il est très important de remarquer que la sécurité de la vieillesse et le supplément de revenu garanti n'influencent pour ainsi dire pas le revenu des femmes des deux secteurs, sauf, le cas échéant, le revenu des femmes du centre. Les résultats du modèle qui tient compte de l'interaction entre les variables retenues et le secteur économique confirment que les femmes de la périphérie sont moins favorisées que les femmes du centre. On peut penser que les régimes de retraite privés n'ont pas le même effet dans les deux secteurs à cause du faible apport des régimes publics au revenu des retraitées de la périphérie. Les femmes du centre tirent un plus grand profit de leur capital humain, et l'augmentation de leur revenu de retraite est plus fortement corrélée avec les revenus qu'elles retirent de leurs placements et d'un REER, de même que des paiements de transfert gouvernementaux. Ces différences de situation pourraient être dues au fait que le travail à temps partiel est plus répandu chez les femmes de la périphérie, mais les données ne permettent pas d'établir jusqu'à quel point. Le temps partiel 
existe aussi chez les femmes du centre et ne peut donc expliquer tous les résultats.

La principale source de revenu déclarée par $63 \%$ des retraitées des industries périphêriques étant leur pension du gouvernement, qui n'est pourtant pas fortement associée avec l'augmentation du revenu de retraite, on conçoit que la plupart vivent sur le seuil de la pauvreté ou en deçà. La situation économique de ces retraitées, plus pénible à tous égards que celle des femmes du centre, illustre les effets de la segmentation du marché du travail et l'impuissance du régime de retraite public devant les inégalités engendrées par ces différences structurelles.

\section{RÉSUMÉ ET CONCLUSION}

Les résultats de ces analyses exploratoires laissent croire que le secteur économique exerce un effet considérable sur le revenu de retraite des Canadiens et que les régimes de retraite publics du Canada sont mal adaptés à cette réalité. Les hommes et les femmes retraités qui ont passé leur vie active dans la périphérie de l'économie sont plus tributaires des pensions gouvernementales et systématiquement, pour toutes les variables de capital humain et de revenu, ils sont plus faiblement rémunérés que les retraités qui travaillaient dans les industries du centre. Le système mixte de protection résultant de la combinaison des régimes publics et des régimes semiprivés de retraite satisfait les besoins des travailleurs privilégiés du centre de l'économie mais crée une sous-classe de retraités parmi les travailleurs qui ont passé la plus grande partie de leur vie active dans le secteur économique secondaire. Le revenu auquel ils donnent droit est surtout destiné aux retraités du centre, à qui il permet de vivre bien au-dessus du seuil de la pauvreté, mais ne fait qu'assurer la subsistance des travailleurs et surtout des travailleuses qui ont occupé des emplois dans le secteur périphérique. Ce système dualiste revêt la forme d'un régime d'aide sociale dans le cas des travailleurs de la périphérie, d'assurance sociale dans celui des travailleurs du centre.

Afin de rendre moins coûteux son système de pensions pour les personnes âgées, le Canada envisage l'institution de deux "super-programmes", l'un de supplément de revenu garanti, qui remplacerait à la fois la sécurité de la vieillesse et le supplément de revenu garanti et comporterait des prestations ajustées 
aux besoins, l'autre d'épargne-retraite, qui serait en quelque sorte un REER basé sur une version universalisée du RPC ou du RRQ (Globe and Mail, 1994 : 11D6). Ce projet, inspiré du modèle "Supplétif", n'augure rien de bon pour les personnes qui n'occupent pas une position avantageuse sur le marché du travail. Il imposerait une responsabilité plus grande aux individus à l'égard de leur sécurité financière au moment de la retraite et, de façon génêrale, ce sont les femmes qui en souffriraient le plus. En particulier, les changements annoncés pénaliseraient lourdement certains hommes et la plupart des femmes du secteur économique périphérique et élargiraient davantage les écarts de revenus entre les Canadiens. Dans cette éventualité, lorsqu'ils se tourneront vers le régime public pour obtenir du soutien, ces groupes feront face à une retraite marquée par la pauvreté, car l'objectif du nouveau système sera de permettre aux retraités de subsister et non, comme auparavant, de maintenir leur niveau de vie et de jouir d'un revenu convenable. L'épargne privée obligatoire instituée par un "Super-REER" et les régimes de retraite privés obligatoires ne résoudraient pas davantage les difficultés de ces travailleurs. La proportion de la population couverte par les régimes privés a décru lentement au fil des années et continuera de diminuer à mesure que l'emploi deviendra plus précaire et que le travail à temps partiel ou temporaire, devenant la nouvelle norme, aura fait oublier les emplois à temps plein assortis de bonnes conditions de travail. De plus, beaucoup de travailleurs de la périphérie n'auront pas les moyens d'adhérer à des régimes privés dont le coût ne pourra qu'augmenter lorsque le secteur privé aura raffermi son emprise sur le domaine des retraites. Étant donné les résultats présentés ici, les autres pays qui envisagent ce type de partage public-privé des responsabilités en matière de protection sociale afin de faire face à la hausse des coûts de la retraite devraient faire preuve de prudence. Il se pourrait bien aussi que les Canadiens souhaitent réexaminer tous les tenants et aboutissants du changement de politique proposé. Celui-ci n'est pas loin de représenter un retour à la loi sur la sécurité de la vieillesse de 1927, qui prévoyait, pour les travailleurs pauvres âgés de 70 ans et plus, un simple revenu de subsistance dont le versement était conditionnel à un examen de la situation financière des intéressés. 


\section{RÉFÉRENCES BIBLIOGRAPHIGUES}

AFIFI, A. A., et V. CLARK, 1990. Computer-Aided Multivariate Analysis. New York, Chapman and Hall, 2e édition.

ATKINSON, A. B., et H. SUTHERLAND, 1993. "Two Nations in Early Retirement? The Case of Britain", dans A. B. ATKINSON et M. REIN, ẽd. Age, Work and Social Security. New York, NY, St. Martin's Press : 132-160.

AVERITT, R. T., 1968. The Dual Economy: The Dynamics of American Industry Structure. New York, Horton.

BECK, E. M., P. M. HORAN et C. M. TOLBERT, 1978. "Stratification in a Dual Economy: A Sectoral Model of Earnings Determination", American Sociological Review, 43 : 704-720.

BECK, S. H., 1983. "Position in the Economic Structure and Unexpected Retirement", Research on Aging, 5, 2 : 197-216.

BECKER, G. S., 1975. Human Capital: A Theoretical and Empirical Analysis with Special Reference to Education. Chicago, University of Chicago Press, 2e édition.

BELGRAVE, L. L., 1988. "The Effects of Race Differences in Work History, Work Attitudes, Economic Resources and Health on Women's Retirement", Research on Aging, 10, 3 : 383-398.

BROWN, R. L., 1991. Economic Security in an Aging Population. Toronto, ON, Butterworths.

CALASANTI, T. M., 1988. "Participation in a Dual Economy and Adjustment to Retirement", International Journal of Aging and Human Development, 26, 1 : 13-27.

CANADIAN INSTITUTE OF ACTUARIES, 1993. Canadian Retirement Income Social Security Programs. Report of the Task Force on Social Security Financing. Ottawa, Canadian Institute of Actuaries.

CANADIAN INSTITUTE OF ACTUARIES, 1995. Troubled Tomorrow's. The Report of The Canadian Institute of Actuaries' Task Force on Retirement Savings. Ottawa, Canadian Institute of Actuaries.

CASEY, B., 1989. "Early Retirement: The Problems of "Instrument Substitution" and "Cost Shifting" and Their Implications for Restructuring the Process of Retirement", dans W. SCHMÄHL, éd. Redefining the Process of Retirement: An International Perspective. Berlin, Allemagne, Springer-Verlag : 133-150.

CHEN, Yung-Ping, 1994. "Equivalent Retirement Ages" and Their Implications for Social Security and Medicare Financing", The Gerontologist, 34, $6: 731-735$.

CHOW, G. C., 1960. "Tests of Equality Between Sets of Coefficients in Two Linear Regressions", Econometrica, $28: 591-605$.

CLAIRMONT, D., R. APOSTLE et R. KRECKEL, 1983. "The Segmentation Perspective as a Middle-Range Conceptualization in Sociology", Canadian Journal of Sociology, 8, $3: 245-271$. 
CONSEIL ECONOMIQUE DU CANADA, 1991. Employment in the Service Economy. Ottawa, Approvisionnements et Services Canada.

DALE, A., et C. BAMFORD, 1988. "Older Workers and the Peripheral Workforce: The Erosion of Gender Differences", Ageing and Society, $8: 43-62$.

DIAMOND, P. A., et J. A. HAUSMAN, 1984. "The Retirement and Unemployment Behavior of Older Men", dans H. J. AARON et G. BURLESS, éd. Retirement and Economic Behavior. Washington, D. C., Brookings Institution : 97-134.

DICKENS, W. T., et K. LANG, 1985. "A Test of Dual Labor Market Theory", American Economic Review, 75 : 792-805.

DOWD, J., 1980. Stratification Among the Aged. Monterey, CA, Brooks/Cole Publishing Company.

EDWARDS, R., 1979. Contested Terrain. New York, Basic Books.

EKERDT, D. J., et S. DEVINEY, 1990. "On Defining Persons as Retired", Journal of Aging Studies, 4, 3: 21 1-229.

FURLONG, A., 1990. "Labour Market Segmentation and The Age Structuring of Employment Opportunities for Young People», Work, Employment and Society, 4, 2 : 253-269.

GHALAM, N. ZUKEWICH, 1994. "Women in the Workplace", dans C. MCKIE et K. THOMPSON, éd. Canadian Social Trends: A Canadian Studies Reader. Toronto, ON, Thompson Educational Publishing, vol. 2 : 141-145.

GIBSON, R. C., 1987. "Reconceptualizing Retirement for Black Americans", The Gerontologist, 27, $6: 691-698$.

GLOBE AND MAIL, 1994. "Toward a Renewed Pension System", Toronto, Globe and Mail, 11 mars : D6.

GRANOVETTER, M., 1984. "Small is Bountiful: Labor Markets and Establishment Size", American Sociological Review, 49 : 323-343.

GUEST, D., 1990. The Emergence of Social Security in Canada. Vancouver, University of British Columbia Press, 2e édition.

GUILLEMARD, A., 1991. "International Perspectives on Early Withdrawal from the Labor Force", dans J. MYLES et J. QUADAGNO, ed. States, Labor Markets, and the Future of OldAge Policy. Philadelphie, PA, Temple University Press : 209-226.

GUILLEMARD, A., et M. REIN, 1993. "Comparative Patterns of Retirement: Recent Trends in Developed Societies", Annual Reviews of Sociology, 19:469-503.

HARDY, M., J. QUADAGNO et W. HOFFMAN, 1993. "Private Pensions, State Regulation and Income Security for Older Workers: The Case of the Auto-Industry", dans M. SHALEV, éd. Occupational Welfare and the Welfare State in Comparative Perspective. New York, Plenum Publishers. 
HAYWARD, M. D., et W. R. GRADY, 1990. "Work and Retirement Among a Cohort of Older Men in the United States, 1966-1983", Demography, 27, 3 : 337-356.

HENDRICKS, J., et C. E. MCALLISTER, 1983. "An Alternative Perspective on Retirement: A Dual Economic Approach", Ageing and Society, $33: 279-299$.

HENRETTA, J. C., A. M. O'RAND et C. G. CHAN, 1993. "Joint Role Investments and Synchronization of Retirement: A Sequential Approach to Couples' Retirement Timing", Social Forces, 71, 4 : 981- 1000 .

HENRETTA, J. C., et R. T. CAMPBELL, 1976. "Status Attainment and Status Maintenance: A Study of Stratification in Old Agen, American Sociological Review, 41 : 981-992.

HOERL, A. E., et R. W. KENNARD, 1970. "Ridge Regression: Application to Non-Orthogonal Problems", Technometrics, 12 : 55-82.

HUGHES, K. D., et G. S. LOWE, 1994. "The Organization of Work", dans W. MELOFF et D. PIERCE, éd. An Introduction to Sociology. Scarborough, ON, Nelson Canada : 124-145.

KRAHN, H. J., et G. S. LOWE, 1993. Work, Industry, and Canadian Society. Scarborough, ON, Thompson Canada, Ltd.

LACZKO, F., et C. PHILLIPSON, 1991. Changing Work and Retirement. Philadelphie, PA, Open University Press.

LEON, J., 1985. "The Effects of Labor Market Segmentation on Economic Resources in Retirement", Social Science Research, 14 : 351-373.

LUSSIER, G., et A. V. WISTER, 1995. "A Study of Workforce Aging of the British Columbia Public Service, 1983-1991", Canadian Journal on Aging, 14, $3: 480-497$.

MAIER, G., et P. WEISS, 1991. "Segmentation, Mobility and the Spatial Distribution of Activities", Labour, 5, 1 : 3-22.

MCDANIEL, S. A., et E. M. GEE, 1993. "Social Policies Regarding Caregiving to Elders: Canadian Contradictions", Journal of Aging and Social Policy, 5 : 1-2.

MCDONALD, L., 1983. The Retirement Spectrum: A Socioeconomic Analysis. Université de Calgary, Département de sociologie, thèse de Ph. D.

MCDONALD, L., et R. A. WANNER, 1992. "Part-time Work and the Older Worker in Canada", dans K. LUNDY, éd. Working Part-Time: Risks and Opportunities. New York, Praeger : 187-204.

MCDONALD, P. L., 1989. "Les travailleuses et travailleurs âgés et l'économie duale», dans H. DAVID, èd. Le Vieillissement au travail: une question de jugement. Montréal, Institut de recherche appliquée sur le travail : 56-62. 
MCDONALD, P. L., et R. A. WANNER, 1984. "Socioeconomic Determinants of Early Retirement in Canadan, Canadian Journal on Aging, 3, 3 : 105-116.

MCDONALD, P. L., et R. A. WANNER, 1989. «Public Policy and the Future of Retirement in Canada". Communication présentée au colloque annuel de la Canadian Association on Gerontology, Ottawa, octobre.

MCDONALD, P. L., et R. A. WANNER, 1990. Retirement in Canada. Toronto, ON, Butterworths Canada Ltd.

MELLOW, W., 1983. "Employer Size, Unionism, and Wages", dans Research in Labor Economics, Supplement 2. Greenwich, CT, JAI Press.

MÉTHOT, S., 1987. "Employment Patterns of Elderly Canadians", Canadian Social Trends, automne : 7-11.

MINISTĖRE DES FINANCES, 1992. Government of Canada: Personal Income Expenditures. Ottawa, Ministère des Finances.

MINISTÈRE DES FINANCES, 1994. Creating a Healthy Fiscal Climate. Ottawa, Ministère des Finances.

MINKLER, M., et C. L. ESTES, éd., 1991. Critical Perspectives on Aging: The Political and Moral Economy of Growing Old. Amityville, NY, Baywood Publishing Company.

MUELLER, C. W., E. MUTRAN et E. BOYLE, 1988. "Age Discrimination in Earnings in a Dual Economy Market". University of Iowa, Département de sociologie, inédit.

MYLES, J., et D. STREET, 1995. "Should the Economic Life Course Be Redesigned? Old Age Security in a Time of Transition", Canadian Journal on Aging, 14, $2: 335-359$.

MYLES, J., et L. TEICHROEW, 1991. "The Politics of Dualism: Pension Policy in Canada", dans J. MYLES et J. QUADAGNO, éd. States, Labor Markets, and the Future of Old-Age Policy. Philadelphie, PA, Temple University Press : 84-104.

NELSON, J. I., 1994. "Work and Benefits: The Multiple Problems of Service Sector Employment", Social Problems, 41, 2 : 240-256.

NIELSEN, F., 1994. "Income Inequality and Industrial Development: Dualism Revisited", American Sociological Review, 59 : 654-677.

OI, W. Y., 1990. "Employment Relations in Dual Labor Markets ("It's Nice Work If You Can Get It")", Journal of Labor Economics, 8, 1 : S124- 149.

O'NEILL, J., 1994. "Changing Occupational Structure", dans C. MCKIE et K. THOMPSON, éd. Canadian Social Trends: A Canadian Studies Reader. Toronto, ON, Thompson Educational Publishing, vol. 2 : 259-263.

O'RAND, A. M., et J. C. HENRETTA, 1982. "Delayed Career Entry, Industrial Pension Structure, and Early Retirement in a Cohort of Unmarried Women", American Sociological Review, 47 : 365-373. 
PALMORE, B., B. BURCHETT, C. FILLENBAUM, L. GEORGE et L. WALLMAN, 1985. Retirement: Causes and Consequences. New York, Springer Publishing Co.

PARNES, H. S., et L. J. LESS, 1985. "The Volume and Pattern of Retirement, 1966-1981", dans H. S. PARNES et al., éd. Retirement Among American Men. Lexington, MA, Lexington Books : 57-77.

PESANDO, J., et M. GUNDERSON, 1988. "Retirement Incentives Contained in Occupational Pension Plans and their Implication for the Mandatory Retirement Debate", Canadian Journal of Economics, 21 : 246-264.

PINEO, P. C., J. PORTER et H. A. MCROBERTS, 1977. "The 1971 Census and the Socioeconomic Classification of Occupations", Canadian Review of Sociology and Anthropology, 14 : 91-102.

PIORE, M. J., 1975. "Notes for a Theory of Labor Market Segmentation", dans R. EDWARDS, M. REICH et D. GORDON, éd. Labor Market Segmentation. Lexington, D. C. Health and Company.

QUADAGNO, J., et J. MYLES, 1991. "Introduction: States, Labor Markets, and the Future of Old-Age Policy", dans J. MYLES et J. QUADAGNO, ed. States, Labor Markets, and the Future of OldAge Policy. Philadelphie, PA, Temple University Press : 3-15.

REITER, E., 1991. Making Fast Food: From the Frying Pan Into the Fryer. Montréal et Kingston, McGill-Queen's University Press.

SAKAMOTO, A., et M. D. CHEN, 1991. "Inequality and Attainment in a Dual Labor Market», American Sociological Review, 56 : 295-308.

SCHELLENBERG, G., 1994. The Road to Retirement: Demographic and Economic Changes in the 90s. Ottawa, Canadian Council on Social Development.

SCHULZ, J. H., 1980. The Economics of Aging. Belmont, CA, Wadsworth.

SCHULZ, J. H., 1991. "Epilogue: The "Buffer Years": Market Incentives and Evolving Retirement Policies", dans J. MYLES et J. QUADAGNO, ed. States, Labor Markets, and the Future of OldAge Policy. Philadelphie, PA, Temple University Press : 295-308.

SHEPPARD, H. L., 1991. "Early Retirement: Questions and Speculations", dans J. MYLES et J. QUADAGNO, èd. States, Labor Markets, and the Future of Old-Age Policy. Philadelphie, PA, Temple University Press : 290-294.

SØRENSEN, A. B., et A. L. KALLEBERG, 1981. "An Outline of a Theory of the Matching of Persons to Jobs", dans I. BERG, éd. Sociological Perspectives on Labor Markets. New York, Academic Press : 49-74.

SPILERMAN, S., 1977. "Careers, Labor Market Structure, and Socioeconomic Achievement", American Journal of Sociology, 83 : $551-593$. 
STATISTIQUE CANADA, 1994. Income Distribution by Size in Canada, 1993. Ottawa, Approvisionnements et Services Canada.

SZINOVACZ, M., D. J. EKERDT et B. H. VINICK, éd., 1992. Families and Retirement. Newbury Park, CA, Sage Publications.

THUROW, L. C., 1975. Generating Inequality: Mechanisms of Distribution in the U.S. Economy. New York, Basic Books.

TIGGES, L. M., 1988. "Age, Earnings, and Change Within the Dual Economy", Social Forces, 66, $3: 676-698$.

TITMUS, R. M., 1955. "Pension Systems and Population Change", Political Quarterly, 26 : 152-166.

TOLBERT, C., P. M. HORAN et E. M. BECK, 1980. «The Structure of Economic Segmentation: A Dual Economy Approach", American Journal of Sociology, 85 : 1095-1116.

VOGT, W. P., 1993. Dictionary of Statistics: A Nontechnical Guide for the Social Sciences. Newbury Park, Sage Publications.

WALKER, A., 1991. "Thatcherism and the New Politics of Old Agen, dans J. MYLES et J. QUADAGNO, éd. States, Labor Markets, and the Future of Old-Age Policy. Philadelphie, PA, Temple University Press : 19-35.

WANNER, R. A., et P. L. MCDONALD, 1986. "The Vertical Mosaic in Later Life: Ethnicity and Retirement in Canada", Journal of Gerontology, 41 : 662-671.

WEITZ, H., 1992. The Pension Promise. Scarborough, Thomson Canada Limited.

WIAL, H., 1991. "Getting a Good Job: Mobility in a Segmented Labor Market», Industrial Relations, 30, $3: 396-416$. 


\title{
RÉSUMÉ-ABSTRACT - RESUMEN
}

\author{
MCDONALD LYNA - LES PENSIONS DE RETRAITE AU CANADA: LES \\ INEGALITÉS D'UN SYSTEMME DUALISTE
}

À cause de leur caractère à la fois public et semi-privé, les politiques sociales canadiennes ont engendré un système dualiste de sécurité du revenu pour les retraités. Certains sont entièrement tributaires des régimes de retraite publics tandis que d'autres bénéficient à la fois de régimes publics et de régimes privés. Cette division se manifeste dans leurs conditions de vie, qui ne sont pas les mēmes suivant qu'ils travaillaient dans le secteur central ou dans le secteur périphérique de l'économie. À l'aide de données provenant de l'Enquête sur le vieillissement et l'autonomie, l'auteur étudie les effets du dualisme économique sur le revenu de retraite des hommes et des femmes de son échantillon $(N=20036)$. Elle compare les retraités du centre et de la périphérie au point de vue de leurs caractéristiques socio-démographiques et de diverses caractéristiques relatives au travail, au revenu et aux dispositions prises pour la retraite. Des modèles de régression lui permettent ensuite de déterminer les facteurs qui influencent le revenu des retraités du centre et de la périphérie. Les résultats tendent à montrer que le système canadien de pensions de retraite accentue la précarité économique des femmes retraitées, dont on sait qu'elle est liée à leur situation sur le marché du travail et à leurs responsabilités familiales. Ce régime dualiste contribue à créer une sous-classe de retraités, et ses principales victimes sont les femmes qui ont passé leur vie active dans la périphérie de l'économie.

\section{MCDONALD Lyn - THE CASUALTIES OF CANADA'S DUALIST PENSION POLICY}

The union of public and semi-private approaches in the formulation of Canadian welfare policy has spawned a dualism in the structure of the income security system for the retired. There are those retirees who depend solely on public pensions and those who benefit from both public and semi-private pensions. This division is reflected in retirement according to the prior location of the worker in the core or peripheral sector of the economy. Using data from the Survey of Ageing and Independence $(N=20,036)$, this research examines the effect of a dual economy on retirement incomes for both men and women. Specifically, the analyses compares core and periphery retirees on sociodemographic characteristics, work characteristics, income characteristics and pension arrangements. Models are then estimated to ascertain what factors influence the retirement incomes for those retirees who previously worked in the core or periphery of the economy. The findings suggest that Canadian pension policy has served to exacerbate the already precarious economic position of retired women, a known outcome of their unstable labour force histories and family responsibilities. The dualist pension system has assisted in producing an underclass of 
retirees, the principal casualties being women who previously worked in the periphery of the economy.

MCDONALD LYNn - LAS PENSIONES DE JUBILACIÓN EN CANADÁ : LAS DESIGUALDADES CAUSADAS POR UN SISTEMA DUALISTA

Siendo a la vez públicas y semi-privadas, las politicas sociales canadienses han fomentado un sistema dualista de seguridad de ingreso para los jubilados. Algunos de éstos dependen por completo de pensiones del sector público, mientras que otros disponen de pensiones a la vez del sector público y del sector privado. Este división se refleja en sus condiciones de vida, que no son las mismas según si estas personas trabajaban en el sector central o en el sector periférico de la economía. Por medio de datos de la Encuesta sobre el envejecimiento y la autonomía, el autor analiza los impactos del dualismo económico sobre los ingresos de jubilación de hombres y mujeres $(N=20,036)$. Se comparan los jubilados del centro y los de la periferia según sus caracteristicas sociodemográficas, y según distintas caracteristicas relativas al trabajo, al ingreso y a los planes de jubilación. Se utilizan luego modelos de regresión para determinar los factores quqe influyen en el ingreso de los jubilados que antes trabajaron en el centro y los de la periferia. Los resultados tienden a demostrar que el sistema canadiense de pensiones de jubilación acentúa la precariedad económica de las mujeres jubiladas, que se sabe es debida a su situación sobre el mercado del trabajo y a sus responsabilidades familiares. Este sistema dualista fomenta la creación de una sub-clase de jubilados, siendo las principales victimas las mujeres que antes laboraron en la periferia de la economia. 